\title{
Isosystolic genus three surfaces critical for slow metric variations
}

\author{
StÉPHANE SABOURAU
}

\begin{abstract}
We show that the two piecewise flat surfaces with conical singularities conjectured by E Calabi as extremal surfaces for the isosystolic problem in genus 3 are critical with respect to some metric variations. The proof relies on a new approach to study isosystolic extremal surfaces.
\end{abstract}

$53 \mathrm{C} 23 ; 53 \mathrm{C} 20,53 \mathrm{C} 22,53 \mathrm{C} 38$

\section{Introduction}

Let $M$ be a non-simply connected closed surface. The systole of a Riemannian metric $g$ on $M$, denoted by sys $(g)$, is defined as the infimum of the lengths of the noncontractible loops of $M$. Define the systolic area of $(M, g)$ as

$$
\sigma(g)=\frac{\operatorname{area}(g)}{\operatorname{sys}(g)^{2}} .
$$

The systolic area of a non-simply connected closed Riemannian surface $(M, g)$ is bounded from below by $2 / \pi$, where equality holds if and only if $(M, g)$ is isometric to a round projective plane; see $\mathrm{Pu}$ [24] and Gromov [15].

Extremal metrics, ie metrics realizing the minimum of the systolic area for a surface of given topological type, are known in only three cases. Around 1949, C Loewner proved that extremal metrics on the torus are flat hexagonal; see Katz [19]. Then, $\mathrm{P} \mathrm{Pu}$ [24] showed that extremal metrics on the projective plane are round. In comparison, C Bavard [4] showed that extremal metrics on the Klein bottle, formed of two round Möbius bands glued together, are not smooth and present a line of singularities.

M Gromov [15] established the existence of extremal metrics in the setting of "generalized metrics". A general study of extremal surfaces has been conducted by E Calabi [11] and pursued by R Bryant [8]. Loosely speaking they showed that extremal metrics tend to have flat regions. E Calabi [11] also described two piecewise flat metrics with conical singularities on the genus three surface extremal in their conformal classes. $\mathrm{He}$ 
conjectured $^{1}$ that these two metrics represent local minima of the systolic area and that one of them corresponds to the global minimum.

The goal of this article is to show that these two piecewise flat metrics are critical for the systolic area with respect to some slow metric variations defined below.

Since the systolic area functional $g \mapsto \sigma(g)$ is not necessarily differentiable, we need to extend the notion of critical point. A similar extension was required by N Nadirashvili [23] and A El Soufi and S Ilias [14] to study Riemannian surfaces maximizing the product $\lambda_{1}(g) \cdot \operatorname{area}(g)$, where $\lambda_{1}(g)$ is the first nonzero eigenvalue of the Laplacian. By analogy with the notion introduced by these authors, we say that a metric $g_{0}$ on $M$ is critical for the systolic area with respect to a metric variation $\left(g_{t}\right)_{t \geq 0}$ if

$$
\sigma\left(g_{t}\right) \geq \sigma\left(g_{0}\right)+o(t) .
$$

We will also need the following.

Definition 1.1 A slow metric variation $\left(g_{t}\right)_{t \geq 0}$ is a one-parameter family of Riemannian metrics $g_{t}$ with conical singularities on $M$ (see Troyanov [27] or Section 12.1) such that the differential of the Busemann function $\beta_{\xi}^{g_{t}}$ in the direction $\xi$ for the metric $g_{t}$ satisfies

$$
\left\|\left(d \beta_{\xi}^{g_{t}}-d \beta_{-\xi}^{g_{t}}\right)-\left(d \beta_{\xi}^{g_{0}}-d \beta_{-\xi}^{g_{0}}\right)\right\|_{L^{2}\left(\widehat{B}_{\xi}\right)}=o(\sqrt{t})
$$

where $\widehat{B}_{\xi}$ is a fundamental domain of the systolic band $B_{\xi}$ in the universal cover of $\left(M, g_{0}\right)$.

The directions $\xi$ involved in this definition correspond to the systolic bands $B_{\xi}$ of the Calabi surface considered. We refer to Definitions 3.1 and 3.2 for precise definitions of the directions $\xi$ and the systolic bands $B_{\xi}$ (which rest on the description of the Calabi surfaces presented in Section 2). We also refer to Section 8 for a definition of Busemann functions.

The condition (1-1) is clearly satisfied if the $L^{2}$-norm of $d \beta_{\xi}^{g_{t}}-d \beta_{\xi}^{g_{0}}$ on $\widehat{B}_{\xi}$ is equal to $o(\sqrt{t})$. We could also use the $L^{1}$-norm in the condition (1-1).

Note that if the differentials of the Busemann functions vary smoothly, or even Lipschitz, with respect to $t$, then the metric variation $\left(g_{t}\right)_{t \geq 0}$ is slow. Actually, the first part of the article does not rely on metric variations, it only occurs in the last two sections.

Examples of slow metric variations are presented in the last section.

\footnotetext{
${ }^{1}$ In a private discussion, he told me that he was less confident now than at the time of the writing of [11] that the metric with the higher systolic area represents a local minimum, though he still believes that the other metric corresponds to the global minimum.
} 
Let $\left(M_{0}, g_{0}\right)$ be one of the two piecewise flat surfaces of genus three with conical singularities conjectured extremal by E Calabi in [11]; see Section 2 for a description. Note that these two singular surfaces are nonpositively curved in the sense of Alexandrov.

We will deal with normalized metrics, that is, metrics with a systole equal to one.

Theorem 1.2 The metric $g_{0}$ on $M_{0}$ is critical for the systolic area with respect to any normalized slow metric variation. That is,

$$
\sigma\left(g_{t}\right) \geq \sigma\left(g_{0}\right)+o(t)
$$

for every normalized slow metric variation $\left(g_{t}\right)_{t \geq 0}$ of the piecewise flat metric $g_{0}$.

Of course, one can ask whether the assumption on the metric variation is always satisfied or can be dropped. To our knowledge, this is still an open question. Actually, it is possible that any metric variation is slow, and that the restriction on the type of metric variations can be relaxed. In any case, this result shows that the two Calabi surfaces have special isosystolic properties. We do not know any other surface of genus greater than one which is critical with respect to slow metric variations.

In spite of the restriction on the metric variations considered, we believe that the point of view developed in this article could be useful in the study of extremal isosystolic properties on surfaces. We present the general framework of the proof below.

The techniques used in this article fit in this contemporary approach of Riemannian geometry which consists of isometrically embedding a Riemannian manifold $M$ into a space of large dimension, such as a Banach or Hilbert space, through the geometry of $M$. This point of view allows us to study the intrinsic geometry of $M$ through the study of its isometric embedding. This approach was developed by Gromov [15], Besson, Courtois and Gallot [7], Ivanov [18] and Burago and Ivanov [9; 10], for instance, to obtain deep results on the geometry of Riemannian manifolds. We borrow several features from these pioneer works.

Thus, contrary to classical techniques available to study extremal isosystolic surfaces (cf Katz [19], Pu [24], Bavard [4; 5; 6], Katz and Sabourau [22] and Horowitz, Usadi Katz and Katz [17], our proof does not rest on the conformal length method nor does it make use of the uniformization theorem. Instead we embed the universal cover of a Riemannian surface $M$ into an infinite dimensional space in an equivariant way using the geometry of the surface. This infinite dimensional space carries some special Euclidean metrics and two-forms along certain directions. These metrics and two-forms are modeled on a given Calabi surface $M_{0}$ so that its embedding into this 
infinite dimensional space preserves some isometric properties. Here, the combinatorics of the systolic loops of $M_{0}$ plays an essential role. Patching the pullbacks of these metrics and two-forms by the embedding of $M$, we construct an auxiliary metric and a two-form on the competing surface $M$. A comparison of the area measures of the different metrics with the area measure of the two-form shows that it is enough to study the first variation of the integral of the two-form associated to the embedding of $M$. In some sense, the proof of the main theorem boils down to an infinitesimal calibration argument and the two-form considered above can be thought of as an "infinitesimally calibrating" form. The restriction on the metric variation occurs only in the final step to differentiate the integral of the infinitesimally calibrating form.

It seems that a local calibration argument could yield a local lower bound on the systolic area of the Calabi surfaces and not merely an infinitesimal one. However the presence of conical singularities on the Calabi surfaces makes the construction of a form which locally calibrates the embedding tricky. Let us emphasize that no form defined globally calibrates the embedding of the first Calabi surface since this surface is not a global minimum of the systolic area.

We also carried out a similar variational study directly on the auxiliary metric without using the infinitesimally calibrating form but the result turned out to be the same. We will not present this study. Instead we decided to develop the infinitesimally calibrating form approach as it lends itself to estimates for Finsler metrics too; cf Ivanov [18] and Burago and Ivanov [9]. In this case, we have to replace the Riemannian metric with an hexagonal metric as in Calabi [11].

In contrast with the genus three surface, we showed in [25] that extremal metrics on the genus two surface are not piecewise flat with conical singularities. In a joint work [22] with M Katz, we found the infimum of the systolic area on nonpositively curved genus two surfaces and described the extremal metrics. These extremal metrics are piecewise flat with conical singularities but are not extremal for the general isosystolic problem. This shows that extremal metrics for the general isosystolic problem on the genus two surface have regions with positive curvature and regions with negative curvature. To be complete with the genus two case, let us mention that the best available systolic inequality on this surface can be found in [21]. It is proved that every metric on the genus two surface has a systolic area greater or equal to the systolic area of the extremal metric on the torus. The same result holds for surfaces of genus at least 20 [20].

Locally extremal and critical metrics have recently been described on the two-sphere by $\mathrm{F}$ Balacheff $[2 ; 3]$ and the author [26] and on compact rank-one symmetric spaces by J-C Álvarez Paiva and F Balacheff [1] for an extended notion of systolic volume. 
We refer to the survey by C Croke and M Katz [12] and the monograph of M Katz [19] for an overview on other aspects of systolic geometry, especially on the notion of stable systole for which extremal metrics have been described in higher dimension.

Acknowledgement The author is grateful to the referee for his or her careful reading and cogent suggestions, which helped improve the presentation of the article, and for pointing out an incorrect statement in a previous version.

\section{Description of critical surfaces}

In this section, we describe the two piecewise flat surfaces of genus three with conical singularities introduced by E. Calabi and arising in Theorem 1.2. This presentation follows the description of [11].

\subsection{Piecewise flat surface modeled on Fermat's quartic}

The first surface is conformal to the Fermat quartic, defined in the complex projective plane $\mathbb{C} P^{2}$ by the homogeneous polynomial equation

$$
x^{4}+y^{4}+z^{4}=0 .
$$

It is modeled on the triangular hyperbolic surface representing the Fermat quartic. This triangular hyperbolic surface is tiled by 32 equilateral hyperbolic triangles with angles equal to $\pi / 4$ and has 12 vertices with 8 equilateral triangles around each of them. These 12 vertices correspond to the points adjoined in the compactification of the quartic, that is to the points $(x, y, z)$ where one of the coordinates is zero and the quotient of the other two is a primitive eighth root of unity.

Replacing the hyperbolic equilateral triangles by Euclidean equilateral triangles gives rise to a piecewise flat surface $M_{0}=\left(M_{0}, g_{0}\right)$ with 12 conical singularities of angle $8 \pi / 3$, normalized to have a systole equal to one; see Figure 1 . The orientationpreserving isometry group of $M_{0}$ is a group of order 96 . Denote by $\mathcal{T}$ the collection of the equilateral flat triangles tiling $M_{0}$.

Every geodesic in the interior of one of the triangles of $\mathcal{T}$ and parallel to any side of this triangle extends as a geodesic and closes after passing through 6 triangles. These closed geodesics are systolic loops of $M_{0}$.

The systolic area of $M_{0}$ is equal to

$$
\sigma\left(M_{0}\right)=\frac{8 \sqrt{3}}{9} \simeq 1.5396
$$




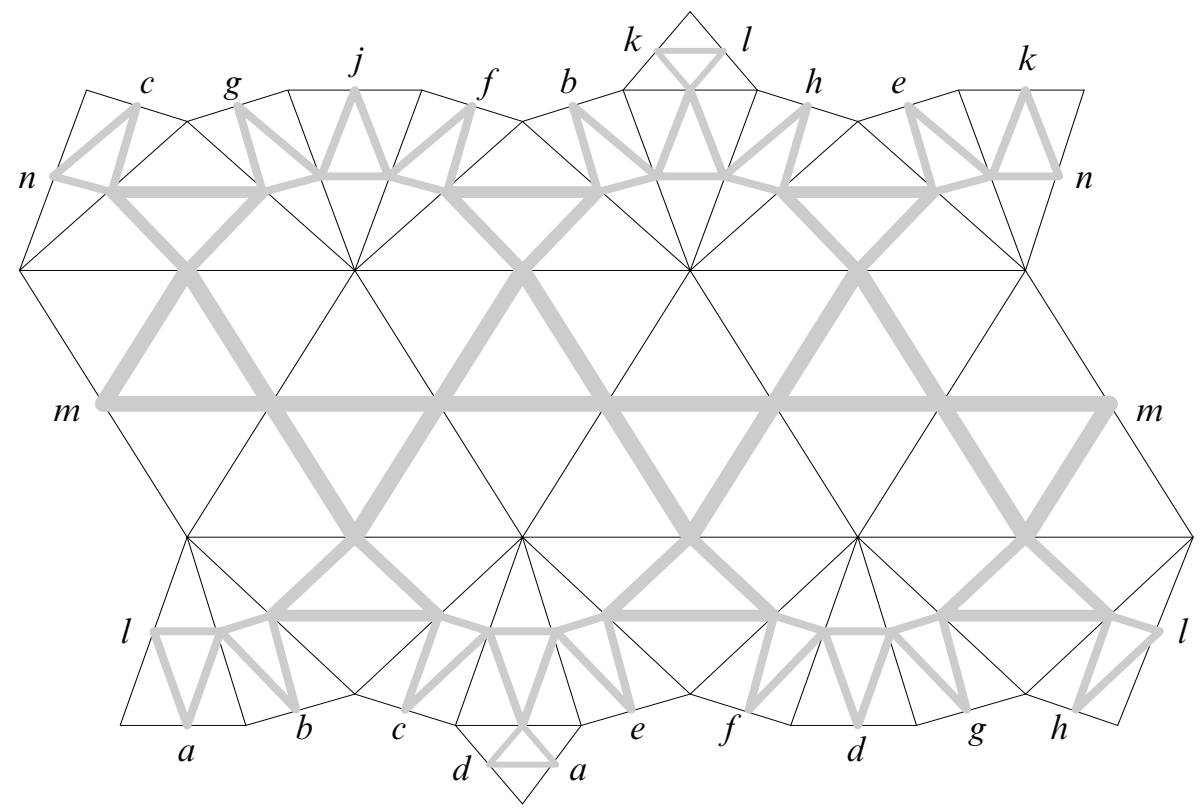

Figure 1

The piecewise flat surface $M_{0}$ can also be described as the polygonal domain of Figure 1 where the sides are identified (preserving the orientation) according to the letters. The 32 equilateral flat triangles of $\mathcal{T}$ are outlined in thin black lines, while the gray stripes, crossing each other pairwise through the midpoint of each edge represent the central portions of the bands foliated by the systolic loops on $M_{0}$. These systolic loops cover the surface $M_{0}$ and induce 32 free homotopy classes. Furthermore, exactly six of them (with the orientation taken into account) pass through every point lying in the interior of a triangle of $\mathcal{T}$.

Note that the conformal class of $M_{0}$ is given by a ramified 4 -fold covering of the octahedron.

\subsection{Piecewise flat surface modeled on Klein's quartic}

The second surface is conformal to the Klein quartic, defined in the complex projective plane $\mathbb{C} P^{2}$ by the homogeneous polynomial equation

$$
x^{3} y+y^{3} z+z^{3} x=0 .
$$

The hyperbolic surface representing the Klein quartic is tiled by 56 equilateral hyperbolic triangles with angles equal to $2 \pi / 7$ and has 24 vertices with 7 equilateral 
triangles around each of them. These 24 vertices correspond to the points adjoined in the compactification of the quartic.

As before, replacing the hyperbolic equilateral triangles by Euclidean equilateral triangles gives rise to a piecewise flat surface $M_{0}=\left(M_{0}, g_{0}\right)$ with 24 conical singularities of angle $7 \pi / 3$, normalized to have a systole equal to one; see Figure 2 for a representation. The orientation-preserving isometry group of $M_{0}$ is isometric to the group $\mathrm{PSL}_{2}\left(\mathbb{F}_{7}\right)$ of order 168 . Denote by $\mathcal{T}$ the collection of the equilateral flat triangles tiling $M_{0}$.

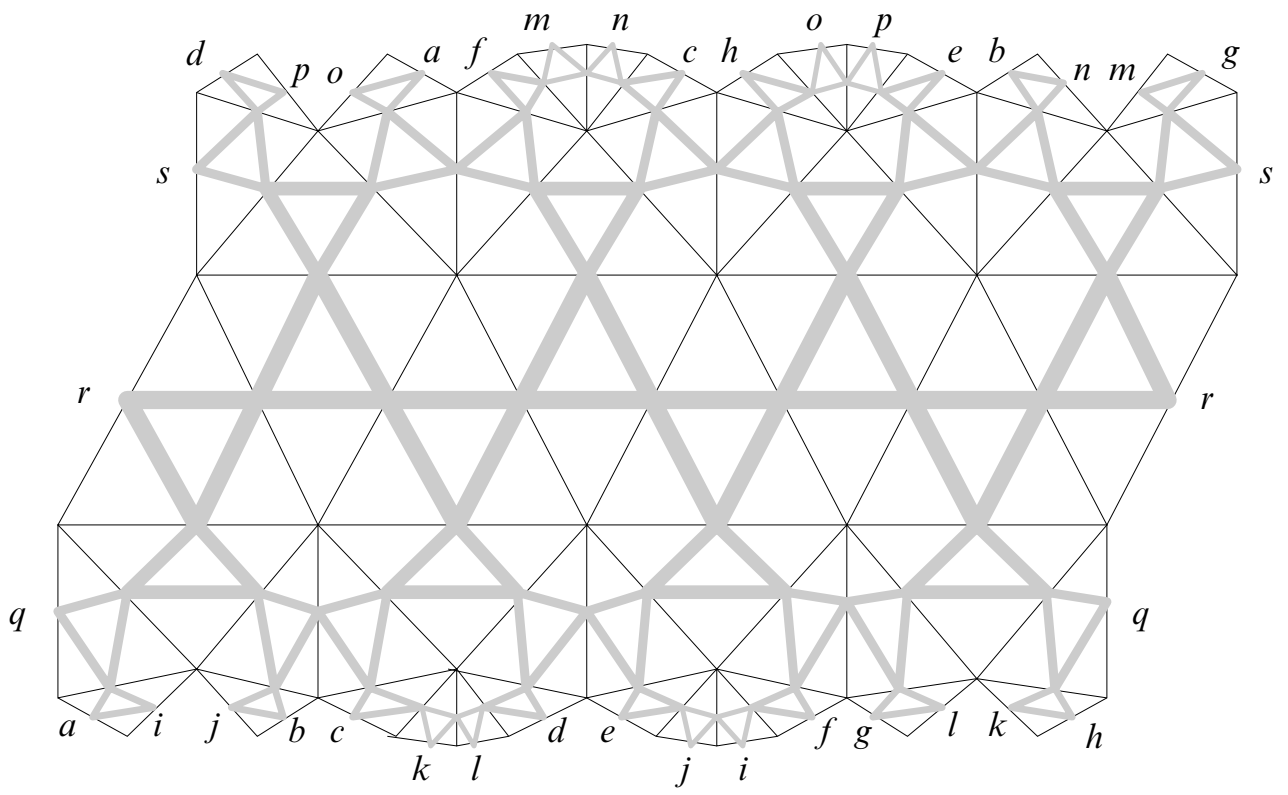

Figure 2

As previously, the systole is achieved by the length of the geodesics parallel to the edges of the triangles of $\mathcal{T}$, which close after passing through 8 triangles. The systolic loops on $M_{0}$ cover the surface and induce 56 free homotopy classes. As before, exactly six of them (with the orientation taken into account) pass through every point lying in the interior of a triangle of $\mathcal{T}$.

The systolic area of $M_{0}$ is equal to

$$
\sigma\left(M_{0}\right)=\frac{7 \sqrt{3}}{8} \simeq 1.5155
$$




\subsection{Comparison with other surfaces}

It might be instructive to compare the systolic areas of the two Calabi surfaces with the ones of surfaces of different topological types.

Given a non-simply connected closed surface $\Sigma$, define $\mathfrak{S}(\Sigma)$ as the infimum of $\sigma(g)$ over all the Riemannian metrics $g$ on $\Sigma$.

The exact value of $\mathfrak{S}$ is known only for the projective plane $\mathbb{R} P^{2}$ [24], the torus $\mathbb{T}^{2}$ [19] and the Klein bottle $\mathbb{K}^{2}[4]$ :

$$
\begin{aligned}
\mathfrak{S}\left(\mathbb{R} P^{2}\right) & =\frac{2}{\pi} \simeq 0.6366 \\
\mathfrak{S}\left(\mathbb{T}^{2}\right) & =\frac{\sqrt{3}}{2} \simeq 0.8660 \\
\mathfrak{S}\left(\mathbb{K}^{2}\right) & =\frac{2 \sqrt{2}}{\pi} \simeq 0.9003
\end{aligned}
$$

In general, we only have

$$
\frac{2}{\pi} \leq \mathfrak{S}(\Sigma)
$$

while for the genus $\gamma$ surfaces $\Sigma_{\gamma}$ the following estimates hold:

$$
\begin{aligned}
\mathfrak{S}\left(\mathbb{T}^{2}\right) \leq \mathfrak{S}\left(\Sigma_{2}\right) & \leq 3(\sqrt{2}-1) \simeq 1.2426 \\
\mathfrak{S}\left(\mathbb{T}^{2}\right) & \leq \mathfrak{S}\left(\Sigma_{\gamma}\right) \text { for every } \gamma \geq 20
\end{aligned}
$$

(see $[24 ; 15 ; 21 ; 22 ; 20])$. Even for the genus three surface, we do not know whether $\mathfrak{S}\left(\mathbb{T}^{2}\right) \leq \mathfrak{S}\left(\Sigma_{3}\right)$.

\section{Systolic directions and admissible maps}

Let us introduce some notation which will be used throughout this article.

Given a genus three surface $M$, denote by $\widetilde{M}$ its universal covering. Denote also by $\Gamma$ the deck transformation group of the universal covering $\widetilde{M}$ of $M$, that is $M \simeq \widetilde{M} / \Gamma$. The deck transformation group $\Gamma$ is a subgroup of $\operatorname{PSL}_{2}(\mathbb{R})$ isomorphic to the fundamental group $\pi_{1}(M)$ of $M$.

Let $M_{0}=\left(M_{0}, g_{0}\right)$ be one of the two piecewise flat Calabi surfaces described in Section 2. Recall that $\operatorname{sys}\left(g_{0}\right)=1$. The metric $g_{0}$ on $M_{0}$ lifts to a metric $\widetilde{g}_{0}$ on $\widetilde{M}_{0}$. Similarly, the natural triangulation $\mathcal{T}$ of $M_{0}$ lifts to a triangulation $\mathcal{T}$ on $\widetilde{M}$. Fix an orientation on $M_{0}$. This orientation induces an orientation on the triangles $\widetilde{\Delta}$ of $\mathcal{T}$ and on their boundaries $\partial \widetilde{\Delta}$. 
Definition 3.1 A systolic direction of $\widetilde{M}_{0}$ is a $\widetilde{g}_{0}$-unit vector of $\widetilde{M}_{0}$ based at the midpoint of a height of an oriented triangle $\widetilde{\Delta}$ of $\mathcal{T}$, parallel to an edge of $\widetilde{\Delta}$ and pointing in the same direction as this edge. The set of systolic directions, denoted by $\mathcal{S}_{0}$, depends on the choice of the surface $M_{0}$ and its orientation. Set $\mathcal{S}_{0}^{*}=$ $\left\{\xi \mid \xi \in \mathcal{S}_{0}\right\} \cup\left\{-\xi \mid \xi \in \mathcal{S}_{0}\right\}$.

A vector $\xi \in \mathcal{S}_{0}^{*}$ induces a geodesic line $c_{\xi}$ on $\widetilde{M}_{0}$ such that $c_{\xi}^{\prime}(0)=\xi$. It also defines a unique element $\alpha_{\xi}$ of $\Gamma \subset \operatorname{PSL}_{2}(\mathbb{R})$, leaving $c_{\xi}$ globally invariant, which takes $c_{\xi}^{\prime}(0)$ to the vector $c_{\xi}^{\prime}(1)$ (recall that $\operatorname{sys}\left(g_{0}\right)=1$ ). Note that the line $c_{\xi}$ projects onto a systolic loop of $M_{0}$ representing $\alpha_{\xi}$.

Two vectors $\xi$ and $\xi^{\prime}$ of $\mathcal{S}_{0}^{*}$ point in the same direction if they generate the same oriented geodesic line up to some time shift. That is,

$$
c_{\xi^{\prime}}(t)=c_{\xi}(t+\tau)
$$

for some $\tau \in \mathbb{R}$ and every $t \in \mathbb{R}$.

Definition 3.2 The systolic band $\widetilde{B}_{\xi}$ of $M_{0}$ induced by a systolic direction $\xi$ of $\widetilde{M}_{0}$ is the union of all triangles of $\mathcal{T}$ through which $c_{\xi}$ passes. The projection $B_{\xi}$ of $\widetilde{B}_{\xi}$ to $M_{0}$, which agrees with the quotient of $\widetilde{B}_{\xi}$ by $\left\langle\alpha_{\xi}\right\rangle$, is also called a systolic band of $M_{0}$.

Definition 3.3 Let us define the infinite product

$$
\mathbb{R}^{\infty}=\prod_{\tilde{\Delta} \in \mathcal{T}} \mathbb{R}_{\tilde{\Delta}}^{3}
$$

where $\mathbb{R}_{\widetilde{\Delta}}^{3}$ is a copy of $\mathbb{R}^{3}$. The space $\mathbb{R}^{\infty}$ is endowed with the product topology.

For every simplex $\widetilde{\Delta}$ of $\mathcal{T}$, number once and for all the systolic directions $\xi_{1}, \xi_{2}, \xi_{3}$ of $\widetilde{M}_{0}$ with basepoints in $\widetilde{\Delta}$ such that the angles between $\xi_{i}$ and $\xi_{i+1}$ are equal to $2 \pi / 3$ (all the indices are taken modulo 3 ).

Every systolic direction $\xi \in \mathcal{S}_{0}$ agrees with one of the three systolic directions $\xi_{i}$ with basepoints in the same triangle $\widetilde{\Delta}_{\xi}$ of $\mathcal{T}$ containing the basepoint of $\xi$.

The $\xi$-coordinate of an element $z$ of $\mathbb{R}^{\infty}$, denoted by $z \xi$, is defined as the $i$-th coordinate of the canonical projection of $z$ in $\mathbb{R}_{\tilde{\Delta}_{\xi}}^{3}$.

The action of $\Gamma$ on $\widetilde{M}_{0}$ induces an action on the (oriented) simplices $\widetilde{\Delta}$ of $\mathcal{T}$, on the systolic directions $\xi$ of $\widetilde{M}_{0}$ and on the vectors of $\mathcal{S}_{0}^{*}$. It also defines an action on $\mathbb{R}^{\infty}$ given by

$$
\gamma \cdot\left(z_{\xi}\right)=\left(z_{\gamma \cdot \xi}\right)
$$


for every $\gamma \in \Gamma$ and $z=\left(z_{\xi}\right) \in \mathbb{R}^{\infty}$. Clearly,

$$
\begin{aligned}
c_{\gamma \cdot \xi} & =\gamma \cdot c_{\xi} \\
\alpha_{\gamma \cdot \xi} & =\gamma \cdot \alpha_{\xi} \cdot \gamma^{-1}
\end{aligned}
$$

for every $\xi \in \mathcal{S}_{0}^{*}$ and $\gamma \in \Gamma$.

Throughout this article, we will consider admissible maps defined as follows.

Definition 3.4 A continuous map $\Phi: \widetilde{M} \rightarrow \mathbb{R}^{\infty}$ is admissible if for every systolic direction $\xi \in \mathcal{S}_{0}$ the following conditions are satisfied:

(1) The map $\Phi_{\xi}: \widetilde{M} \rightarrow \mathbb{R}$ defined as the $\xi$-coordinate map of $\Phi$, ie $\Phi_{\xi}=\Phi(.)_{\xi}$, is differentiable almost everywhere on $\widetilde{M}$.

(2) The one-forms $d \Phi_{\xi}$ and $d \Phi_{\xi}$ defined almost everywhere on $\widetilde{M}$ agree whenever the systolic directions $\xi$ and $\xi^{\prime}$ point in the same direction. That is,

$$
d \Phi_{\xi^{\prime}}=d \Phi_{\xi} .
$$

(3) The one-form $d \Phi_{\xi}$ defined almost everywhere on $\widetilde{M}$ satisfies the following equivariance property

$$
\gamma^{*}\left(d \Phi_{\gamma . \xi}\right)=d \Phi_{\xi}
$$

for every $\gamma \in \Gamma$.

(4) The map $\Phi_{\xi}$ passes to the quotient and induces a map

$$
\overline{\Phi_{\xi}}: \widetilde{M} /\left\langle\alpha_{\xi}\right\rangle \rightarrow \mathbb{R} / \mathbb{Z}
$$

where $\left\langle\alpha_{\xi}\right\rangle$ is the subgroup of $\Gamma$ generated by $\alpha_{\xi}$; cf Definition 3.1.

\section{Riemannian metrics induced by admissible maps}

Let $\widetilde{\Delta}$ be a simplex of $\mathcal{T}$, and $\xi_{1}, \xi_{2}$ and $\xi_{3}$ be the three systolic directions with basepoints in $\widetilde{\Delta}$; cf Definition 3.3. Recall that the angles between $\xi_{i}$ and $\xi_{i+1}$ are equal to $2 \pi / 3$ (all the indices are taken modulo 3 ).

Define a linear projection $\pi_{\tilde{\Delta}}$ as

$$
\begin{aligned}
\pi_{\tilde{\Delta}}: \mathbb{R}^{\infty} & \rightarrow \mathbb{R}_{\widetilde{\Delta}}^{3} \simeq \mathbb{R}^{3} \\
z & \mapsto\left(z_{\xi_{1}}, z_{\xi_{2}}, z \xi_{3}\right) .
\end{aligned}
$$

Let $\Phi: \widetilde{M} \rightarrow \mathbb{R}^{\infty}$ be an admissible map. Denote by $\Phi_{i}$ the $\xi_{i}$-coordinate map $\Phi_{\xi_{i}}$ of $\Phi$. Even though $\Phi$ is not necessarily differentiable (no norm has even been set on $\mathbb{R}^{\infty}$ ), the composite $\pi_{\tilde{\Delta}^{\circ}}^{\circ} \Phi$, which sends $x$ to $\left(\Phi_{i}(x)\right)_{i=1}^{3}$, is differentiable almost everywhere on $\widetilde{M}$. This allows us to define a metric $g_{\Phi}$ on $M$ as follows. 
Definition 4.1 Let $\widetilde{\Delta} \in \mathcal{T}$. Denote by $\tilde{g}_{\Phi}$ the pullback on $\widetilde{\Delta}$ of the standard Euclidean metric $g_{E}$ of $\mathbb{R}^{3}$ by $\left.\pi_{\tilde{\Delta}} \circ \Phi\right|_{\tilde{\Delta}}$. That is,

$$
\tilde{g}_{\Phi}=\left(\left.\pi_{\tilde{\Delta}} \circ \Phi\right|_{\tilde{\Delta}}\right)^{*}\left(g_{E}\right)
$$

where $\left.\Phi\right|_{\tilde{\Delta}}$ is the restriction of $\Phi$ to $\widetilde{\Delta}$. Strictly speaking, the quadratic form $\widetilde{g}_{\Phi}$, which is only defined almost everywhere on $\widetilde{\Delta}$, can be degenerate (as the differential of $\left.\pi_{\tilde{\Delta}} \circ \Phi\right)$. In spite of that, we will still refer to $\widetilde{g}_{\Phi}$ as a metric. Putting together the metrics $\widetilde{g}_{\Phi}$ on each simplex $\widetilde{\Delta}$ of $\mathcal{T}$ gives rise to a metric on $\widetilde{M}$ still denoted by $\widetilde{g}_{\Phi}$.

Note that the metric $\tilde{g}_{\Phi}$ is not well defined on the one-skeleton of the triangulation $\mathcal{T}$ since the metrics defined on each simplex of $\mathcal{T}$ do not necessarily agree on their common edges.

Definition 4.2 Two maps $\varphi, \psi: X \rightarrow \mathbb{R}^{3}$ are said to agree up to a coordinate permutation of $\mathbb{R}^{3}$ if there exists a permutation $\sigma$ of $\{1,2,3\}$ such that for every $x \in X$ and $i \in\{1,2,3\}$, the coordinates $\varphi(x)_{i}$ and $\psi(x)_{i}$ of $\varphi(x)$ and $\psi(x)$ satisfy $\varphi(x)_{\sigma(i)}=\psi(x)_{i}$. In this case, we write $\varphi \simeq \psi$. Note that the permutation $\sigma$ does not depend on $x$.

This definition extends to vectors of $\mathbb{R}^{3}$ viewed as constant maps in $\mathbb{R}^{3}$.

Let us state a result that will be used to establish some invariance properties a couple of times in this article.

Lemma 4.3 Let $\tilde{\Delta} \in \mathcal{T}$ and $\gamma \in \Gamma$. Then the two maps

$$
\gamma^{*}\left[d\left(\left.\pi_{\gamma \cdot \tilde{\Delta}} \circ \Phi\right|_{\gamma \cdot \tilde{\Delta}}\right)\right] \quad \text { and } \quad d\left(\left.\pi_{\tilde{\Delta}} \circ \Phi\right|_{\tilde{\Delta}}\right)
$$

agree almost everywhere on $\Delta$ up to a coordinate permutation of $\mathbb{R}^{3}$. That is,

$$
\gamma^{*}\left[d\left(\left.\pi_{\gamma . \tilde{\Delta}^{\circ}} \circ \Phi\right|_{\gamma . \tilde{\Delta}}\right)\right] \simeq d\left(\left.\pi_{\tilde{\Delta}} \circ \Phi\right|_{\widetilde{\Delta}}\right) .
$$

Proof Let $\left(\xi_{i}\right)_{i=1}^{3}$ and $\left(\xi_{i}^{\prime}\right)_{i=1}^{3}$ be the three systolic directions of $\widetilde{M}_{0}$ with basepoints in $\widetilde{\Delta}$ and $\gamma . \widetilde{\Delta}$; cf Definition 3.3. Since $\gamma$ is an orientation preserving isometry of $\widetilde{M}_{0}$, it preserves the systolic directions of $\widetilde{M}_{0}$. Hence, $\gamma .\left(\xi_{i}\right)_{i=1}^{3} \simeq\left(\xi_{i}^{\prime}\right)_{i=1}^{3}$. Thus,

$$
\begin{aligned}
\left.\pi_{\gamma \cdot \tilde{\Delta}} \circ \Phi\right|_{\gamma . \tilde{\Delta}} & =\left(\left.\Phi_{\xi_{i}^{\prime}}\right|_{\gamma \cdot \tilde{\Delta}}\right)_{i=1}^{3} \\
& \simeq\left(\left.\Phi_{\gamma \cdot \xi_{i}}\right|_{\gamma \cdot \tilde{\Delta}}\right)_{i=1}^{3} .
\end{aligned}
$$

Differentiating this relation yields

$$
d\left(\left.\pi_{\gamma . \tilde{\Delta}} \circ \Phi\right|_{\gamma . \tilde{\Delta}}\right) \simeq\left(\left.d \Phi_{\gamma \cdot \xi_{i}}\right|_{\gamma . \tilde{\Delta}}\right)_{i=1}^{3} .
$$


From Definition 3.4 (3), we derive

$$
\begin{aligned}
\gamma^{*}\left(\left.d \Phi_{\gamma \cdot \xi_{i}}\right|_{\gamma \cdot \tilde{\Delta}}\right)_{i=1}^{3} & =\left(\left.d \Phi_{\xi_{i}}\right|_{\widetilde{\Delta}}\right)_{i=1}^{3} \\
& =d\left(\left.\pi_{\widetilde{\Delta}} \circ \Phi\right|_{\widetilde{\Delta}}\right) .
\end{aligned}
$$

Hence, $\gamma^{*}\left[d\left(\left.\pi_{\gamma . \tilde{\Delta}^{\circ}} \circ\right|_{\gamma . \tilde{\Delta}}\right)\right] \simeq d\left(\left.\pi_{\tilde{\Delta}} \circ \Phi\right|_{\tilde{\Delta}}\right)$.

The following result shows that the metric $\tilde{g}_{\Phi}$ descends to $M$.

Lemma 4.4 The metric $\widetilde{g}_{\Phi}$ is $\Gamma$-invariant on $\widetilde{M}$.

Proof Let $\gamma \in \Gamma$ and $x \in \widetilde{M}$ such that $\widetilde{g}_{\Phi}$ and $\gamma^{*} \widetilde{g}_{\Phi}$ are defined at $x$. Denote by $\widetilde{\Delta}$ the simplex of $\mathcal{T}$ such that $x \in \widetilde{\Delta}$. We have

$$
\begin{aligned}
& \left.\left(\gamma^{*} \widetilde{g}_{\Phi}\right)\right|_{x}=\left.\left[\gamma^{*}\left(\pi_{\gamma . \tilde{\Delta}^{\circ}} \circ \Phi\right)^{*} g_{E}\right]\right|_{x} \\
& =\left.\left[\gamma^{*} d\left(\pi_{\gamma \cdot \tilde{\Delta}^{\circ}} \circ\right)\right]^{*}\right|_{x} g_{E} .
\end{aligned}
$$

From Lemma 4.3 and since $g_{E}$ is invariant by coordinate permutations, we derive

$$
\begin{aligned}
\left.\left(\gamma^{*} \tilde{g}_{\Phi}\right)\right|_{x} & =\left.d\left(\pi_{\tilde{\Delta}^{\circ}} \circ \Phi\right)^{*}\right|_{x} g_{E} \\
& =\left.\left[\left(\pi_{\widetilde{\Delta}} \circ \Phi\right)^{*} g_{E}\right]\right|_{x} \\
& =\left.\tilde{g}_{\Phi}\right|_{x} .
\end{aligned}
$$

Hence, $\gamma^{*} \tilde{g}_{\Phi}=\tilde{g}_{\Phi}$.

Definition 4.5 The metric induced by $\tilde{g}_{\Phi}$ on the quotient $M$ will be denoted by $g_{\Phi}$.

\section{Two-forms induced by admissible maps}

Let $\Phi: \widetilde{M} \rightarrow \mathbb{R}^{\infty}$ be an admissible map. As in Section 4, we can define a two-form $\omega_{\Phi}$ on $M$.

Definition 5.1 Assume $\tilde{\Delta} \in \mathcal{T}$. Denote by $\widetilde{\omega}_{\Phi}$ the pullback on $\widetilde{\Delta}$ of the twoform $\sum_{i=1}^{3} d x_{i} \wedge d x_{i+1}$ of $\mathbb{R}^{3}$ by $\left.\pi_{\tilde{\Delta}^{\circ}} \circ\right|_{\tilde{\Delta}}$ (all indices are taken modulo 3 ). That is,

$$
\tilde{\omega}_{\Phi}=\left(\left.\pi_{\tilde{\Delta}} \circ \Phi\right|_{\tilde{\Delta}}\right)^{*}\left(\sum_{i=1}^{3} d x_{i} \wedge d x_{i+1}\right) .
$$

As previously, this two-form is only defined almost everywhere on $\widetilde{\Delta}$. Putting together the two-forms $\widetilde{\omega}_{\Phi}$ on each simplex $\widetilde{\Delta}$ gives rise to a two-form on $\widetilde{M}$ still denoted by $\tilde{\omega}_{\Phi}$. 
The following result shows that the two-form $\widetilde{\omega}_{\Phi}$ descends to $M$.

Lemma 5.2 The one-form $\widetilde{\omega}_{\Phi}$ is $\Gamma$-invariant on $\widetilde{M}$.

Proof The proof proceeds as in the proof of Lemma 4.4: simply replace $\widetilde{g}_{\Phi}$ by $\widetilde{\omega}_{\Phi}$ and $g_{E}$ by $\sum_{i=1}^{3} d x_{i} \wedge d x_{i+1}$. As previously, the result follows from Lemma 4.3 and the invariance of $\sum_{i=1}^{3} d x_{i} \wedge d x_{i+1}$ by coordinate permutations.

Definition 5.3 The two form induced by $\widetilde{\omega}_{\Phi}$ on the quotient $M$ will be denoted by $\omega_{\Phi}$.

\section{Comparison of the area forms $d g_{\Phi}$ and $\omega_{\Phi}$}

Lemma 6.1 Let $\Phi: \widetilde{M} \rightarrow \mathbb{R}^{\infty}$ be an admissible map. Then we have

$$
\left|\omega_{\Phi}\right| \leq \sqrt{3} d g_{\Phi}
$$

almost everywhere on $M$, where $\left|\omega_{\Phi}\right|$ is the area measure of $\omega_{\Phi}$ on $M$ and $d g_{\Phi}$ is the area measure of $g_{\Phi}$ on $M$.

Proof Let $\widetilde{\Delta} \in \mathcal{T}$. The two-form $\widetilde{\omega}_{\Phi}$ is represented on $\widetilde{\Delta}$ by the sum

$$
\sum_{i=1}^{3} d \Phi_{i} \wedge d \Phi_{i+1}
$$

where $\Phi_{i}=\Phi_{\xi_{i}}$ and the $\xi_{i}$ 's are the three systolic directions with basepoints in $\widetilde{\Delta}$; cf Definition 3.3.

Let $x \in \widetilde{\Delta}$ such that the $\Phi_{i}$ 's are differentiable at $x$ for $i \in\{1,2,3\}$. Each bivector $d_{x} \Phi_{i} \wedge d_{x} \Phi_{i+1}$ of the cotangent plane $T_{x}^{*} \widetilde{M}$ can be represented by the oriented $\tilde{g}_{0}$-area of the parallelogram $\#_{i}$ spanned by the $\tilde{g}_{0}$-gradients $\nabla_{x} \Phi_{i}$ and $\nabla_{x} \Phi_{i+1}$ of $\Phi_{i}$ and $\Phi_{i+1}$ at $x[18 ; 9]$ so that

$$
\tilde{\omega}_{\Phi}=\sum_{i=1}^{3} \operatorname{area}\left(\#_{i}\right) d \widetilde{g}_{0} .
$$

Note that

$$
\operatorname{area}\left(\#_{i}\right)=\operatorname{det}_{\tilde{g}_{0}}\left(\nabla_{x} \Phi_{i}, \nabla_{x} \Phi_{i+1}\right) .
$$

On the other hand, the differential of $\pi_{\widetilde{\Delta}} \circ \Phi$ at $x$ is given by

$$
\begin{aligned}
d_{x}\left(\pi_{\tilde{\Delta}} \circ \Phi\right): T_{x} \widetilde{M}_{0} & \rightarrow \mathbb{R}^{3} \\
u & \mapsto\left(\left\langle\nabla_{x} \Phi_{i}, u\right\rangle\right)_{i=1}^{3} .
\end{aligned}
$$


Let $\left(e_{1}, e_{2}\right)$ be a positively oriented $\tilde{g}_{0}$-orthonormal basis of $T_{x} \widetilde{M}_{0}$. The crossproduct of $d_{x}\left(\pi_{\tilde{\Delta}} \circ \Phi\right)\left(e_{1}\right)$ and $d_{x}\left(\pi_{\tilde{\Delta}} \circ \Phi\right)\left(e_{2}\right)$ is the vector of $\mathbb{R}^{3}$ given by

$$
d_{x}\left(\pi_{\widetilde{\Delta}} \circ \Phi\right)\left(e_{1}\right) \times d_{x}\left(\pi_{\widetilde{\Delta}} \circ \Phi\right)\left(e_{2}\right)=\left(\operatorname{det}_{\widetilde{g}_{0}}\left(\nabla_{x} \Phi_{i+1}, \nabla_{x} \Phi_{i+2}\right)\right)_{i=1}^{3}
$$

where the index $i$ is taken modulo 3. Now, recall that $\widetilde{g}_{\Phi}=\left(\pi_{\tilde{\Delta}} \circ \Phi\right)^{*} g_{E}$. Hence,

$$
\begin{aligned}
d \widetilde{g}_{\Phi} & =\left\|d_{x}\left(\pi_{\tilde{\Delta}} \circ \Phi\right)\left(e_{1}\right) \times d_{x}\left(\pi_{\tilde{\Delta}} \circ \Phi\right)\left(e_{2}\right)\right\|_{E} d \widetilde{g}_{0} \\
& =\sqrt{\sum_{i=1}^{3} \operatorname{area}\left(\#_{i}\right)^{2}} d \widetilde{g}_{0}
\end{aligned}
$$

from Equations (6-3) and (6-2). By the Cauchy-Schwarz inequality, we derive

$$
\widetilde{\omega}_{\Phi}=\sum_{i=1}^{3} \operatorname{area}\left(\#_{i}\right) d \widetilde{g}_{0} \leq \sqrt{3} \sqrt{\sum_{i=1}^{3} \operatorname{area}\left(\#_{i}\right)^{2}} d \widetilde{g}_{0}=\sqrt{3} d \widetilde{g}_{\Phi} .
$$

\section{Area measures of 1-Lipschitz admissible maps}

Let $g$ be a metric on $M$ and $\widetilde{g}$ its lift on $\widetilde{M}$. Even though the supremum norm $\|\cdot\|_{\infty}$ is not always finite on $\mathbb{R}^{\infty}$, the following definition of Lipschitz maps for the supremum norm still makes sense.

Definition 7.1 A map $\Phi: \widetilde{M} \rightarrow \mathbb{R}^{\infty}$ is 1 -Lipschitz if

$$
\|\Phi(x)-\Phi(y)\|_{\infty} \leq d_{\widetilde{g}}(x, y)
$$

for every $x, y \in \widetilde{M}$, or equivalently if $\Phi_{\xi}$ is 1 -Lipschitz as a function with real values for every $\xi \in \mathcal{S}_{0}$.

With this notion, we can state the following result.

Lemma 7.2 Let $\Phi: \widetilde{M} \rightarrow \mathbb{R}^{\infty}$ be a 1-Lipschitz admissible map. Then we have

$$
d g_{\Phi} \leq \frac{3}{2} d g
$$

almost everywhere on $M$, where $d g$ and $d g_{\Phi}$ represent the area measures of $g$ and $g_{\Phi}$ on $M$.

Furthermore, equality holds if and only if $g_{\Phi}=\frac{3}{2} g$ almost everywhere on $M$. 
Proof Let $x \in \widetilde{M}$ such that $\widetilde{g}_{\Phi}$ is defined at $x$. Denote by $\widetilde{\Delta}$ the simplex of $\mathcal{T}$ such that $x \in \tilde{\Delta}$. Let $\left(\xi_{i}\right)_{i=1}^{3}$ be the three systolic directions with basepoints in $\tilde{\Delta}$; cf Definition 3.3. We will write $\Phi_{i}=\Phi_{\xi_{i}}$.

Let $\left(e_{1}, e_{2}\right)$ be a positively oriented $\widetilde{g}$-orthonormal basis of $T_{x} \widetilde{M}$. We have

$$
d \widetilde{g}_{\Phi}=\sqrt{\operatorname{det}_{\tilde{g}} \widetilde{g}_{\Phi}} d \widetilde{g}
$$

where $\operatorname{det}_{\tilde{g}} \widetilde{g}_{\Phi}=\left(\operatorname{det} \widetilde{g}_{\Phi}\left(e_{i}, e_{j}\right)\right)_{i, j \in\{1,2\}}$ defines the determinant of $\tilde{g}_{\Phi}$ with respect to $\tilde{g}$. (This definition does not depend on the choice of the positively oriented $\tilde{g}-$ orthonormal basis $\left(e_{1}, e_{2}\right)$.)

The trace of $\left.\tilde{g}_{\Phi}\right|_{x}$ with respect to $\widetilde{g}$ is given by

$$
\begin{aligned}
\operatorname{tr}_{\tilde{g}}\left(\left.\widetilde{g}_{\Phi}\right|_{x}\right) & =\left.\sum_{j=1}^{2}\left(\pi_{\tilde{\Delta}} \circ \Phi\right)^{*}\left(g_{E}\right)\right|_{x}\left(e_{j}, e_{j}\right) \\
& =\sum_{j=1}^{2}\left\|d_{x}\left(\pi_{\tilde{\Delta}} \circ \Phi\right)\left(e_{j}\right)\right\|_{E}^{2} \\
& =\sum_{j=1}^{2} \sum_{i=1}^{3}\left|d_{x} \Phi_{i}\left(e_{j}\right)\right|^{2} \\
& =\sum_{i=1}^{3}\left\|d_{x} \Phi_{i}\right\|_{\tilde{g}}^{2}
\end{aligned}
$$

By assumption, the map $\Phi$ is 1-Lipschitz. Thus, the map $\Phi_{\xi}$ is 1-Lipschitz for every systolic direction $\xi$. Therefore, $\left\|d_{x} \Phi_{i}\right\|_{\tilde{g}} \leq 1$. Hence,

$$
\operatorname{tr}_{\tilde{g}}\left(\widetilde{g}_{\Phi}\right) \leq 3 .
$$

From the geometric-arithmetic mean inequality, we have

$$
\sqrt{\operatorname{det}_{\tilde{g}} \widetilde{g}_{\Phi}} \leq \frac{3}{2}
$$

The result follows from (7-1).

Corollary 7.3 Under the same assumption as Lemma 7.2, we have

$$
\operatorname{area}\left(g_{\Phi}\right) \leq \frac{3}{2} \operatorname{area}(g)
$$

with equality if and only if $g_{\Phi}=\frac{3}{2} g$ almost everywhere. 


\section{Admissible map induced by a metric on $M$}

Fix a Calabi surface $M_{0}$; cf Section 2. Let $g$ be a metric on $M$ and $\widetilde{g}$ be the induced metric on the universal covering $\widetilde{M}$ of $M$.

Given $\xi \in \mathcal{S}_{0}^{*}$ (cf Definition 3.1), we define $\operatorname{sys}_{g}\left(\alpha_{\xi}\right)$ as the least length of a loop of $M$ representing $\alpha_{\xi}$. That is,

$$
\operatorname{sys}_{g}\left(\alpha_{\xi}\right)=\operatorname{sys}\left(\widetilde{M} /\left\langle\alpha_{\xi}\right\rangle\right)
$$

where $\widetilde{M} /\left\langle\alpha_{\xi}\right\rangle$ is endowed with the metric induced by $g$.

Lemma 8.1 There exists a constant $C=C(g)$ such that for every vector $\xi \in \mathcal{S}_{0}^{*}$ (cf Definition 3.1), we have

$$
\left|d_{\widetilde{g}}\left(c_{\xi}(0), c_{\xi}(t)\right)-t \operatorname{sys}_{g}\left(\alpha_{\xi}\right)\right| \leq C .
$$

Proof Let $m=E(t)$ be the integer part of $t$. The $\widetilde{g}$-distance between $c_{\xi(m)}$ and $c_{\xi}(t)$ is uniformly bounded from above.

Let $\gamma$ be a $g$-length minimizing loop in $M$ representing $\alpha_{\xi}$. Denote by $\overline{c_{\xi}}(0)$ the projection of $c_{\xi}(0)$ to $M$ and by $c$ a segment joining $\overline{c_{\xi}}(0)$ to $\gamma$. The loop $c \cup \gamma^{m} \cup c^{-1}$ represents $\alpha_{\xi}^{m}$ and lifts to an arc of $\widetilde{M}$ with endpoints $c_{\xi}(0)$ and $c_{\xi}(m)$. Thus,

$$
d_{\widetilde{g}}\left(c_{\xi}(0), c_{\xi}(m)\right) \leq m \operatorname{sys}_{g}\left(\alpha_{\xi}\right)+2 \operatorname{diam}(g) .
$$

On the other hand, every arc with endpoints $c_{\xi}(0)$ and $c_{\xi}(m)$ projects to a loop of $M$ representing $\alpha_{\xi}^{m}$. From [16, Lemma 4.32], we obtain

$$
m \operatorname{sys}_{g}\left(\alpha_{\xi}\right) \leq d_{\widetilde{g}}\left(c_{\xi}(0), c_{\xi}(m)\right) .
$$

Combined with some triangular inequalities, the bounds (8-3) and (8-4) yield the desired estimate.

The bound (8-2) shows that the following limit-sup (8-5) is finite.

Definition 8.2 Every vector $\xi \in \mathcal{S}_{0}^{*}$ induces a Busemann function $\beta_{\xi}^{g}: \widetilde{M} \rightarrow \mathbb{R}$ with respect to $g$ defined as

$$
\beta_{\xi}^{g}(x)=\limsup _{t \rightarrow \infty} d_{\widetilde{g}}\left(x, c_{\xi}(t)\right)-t \operatorname{sys}_{g}\left(\alpha_{\xi}\right)
$$

where $x \in \widetilde{M}$. Note that $\beta_{\xi}^{g}\left(c_{\xi}(0)\right)=0$.

The function $\beta_{\xi}^{g}$ is $1-$ Lipschitz as a limit-sup of 1-Lipschitz functions. Thus, it is differentiable almost everywhere by the Rademacher theorem. 
Suppose that $\beta_{\xi}^{g}$ is differentiable at $x \in \widetilde{M}$. The $g$-geodesic ray $\gamma$ arising from the $g$-gradient $\nabla_{x} \beta_{\xi}^{g}$ of $\beta_{\xi}^{g}$ at $x$ satisfies the relation

$$
\beta_{\xi}^{g}(\gamma(s))-\beta_{\xi}^{g}(\gamma(0))=s
$$

for every $s \geq 0$. Furthermore, $\beta_{\xi}^{g}$ is differentiable at every point of $\gamma$ and

$$
\nabla_{x} \beta_{\xi}^{g}=\gamma^{\prime}(s)
$$

The relation (8-6) also implies that the projection of $\gamma$ to $M$ is a geodesic curve without transverse self-intersecting points.

Definition 8.3 Let us define a map $\Phi^{g}: \widetilde{M} \rightarrow \mathbb{R}^{\infty}$ as follows.

As previously, given a vector $\xi \in \mathcal{S}_{0}^{*}$, we define $\phi_{\xi}^{g}: \widetilde{M} \rightarrow \mathbb{R}$ as

$$
\phi_{\xi}^{g}(x)=\frac{1}{2}\left(\beta_{\xi}^{g}(x)-\beta_{-\xi}^{g}(x)\right)
$$

for every $x \in \widetilde{M}$. Note that $\phi_{-\xi}^{g}=-\phi_{\xi}^{g}$.

Given $\xi \in \mathcal{S}_{0}^{*}$, denote by $\xi(i)$ with $i \in \mathbb{Z}$ the vectors of $\mathcal{S}_{0}^{*}$ pointing in the same direction as $\xi$. More precisely, $\xi(i)=c_{\xi}^{\prime}(i / n)$, where $n$ is equal to 6 if $M_{0}$ is the first Calabi surface (cf Section 2.1), and to 8 if $M_{0}$ is the second Calabi surface; cf Section 2.2 (recall that $\operatorname{sys}\left(M_{0}\right)=1$ ). Note that $\xi(0)=\xi, \xi(n)=\alpha_{\xi} \cdot \xi$, $\xi(i)(j)=\xi(i+j)$ and $\alpha_{\xi(i)}=\alpha_{\xi}$.

We can now define a function $\Phi_{\xi}^{g}: \widetilde{M} \rightarrow \mathbb{R}$ as

$$
\Phi_{\xi}^{g}(x)=\left(\frac{1}{n} \sum_{i=0}^{n-1} \phi_{\xi(i)}^{g}(x)\right) / \operatorname{sys}_{g}\left(\alpha_{\xi}\right)
$$

for every $x \in \widetilde{M}$ and every vector $\xi \in \mathcal{S}_{0}^{*}$. Note that $\Phi_{-\xi}^{g}=-\Phi_{\xi}^{g}$.

The functions $\Phi_{\xi}^{g}$ give rise to a map $\Phi^{g}: \widetilde{M} \rightarrow \mathbb{R}^{\infty}$.

Example 8.4 Let $g=g_{0}$ and $\xi \in \mathcal{S}_{0}$ be a systolic direction on $M_{0}$. Denote by $\widetilde{\Delta}$ the flat triangle of $\mathcal{T}$ where the basepoint of $\xi$ lies in. The Busemann function $\beta_{\xi}^{g}$ is differentiable on $\widetilde{\Delta}$ and its $\widetilde{g}_{0}$-gradient on $\widetilde{\Delta}$ is a unit vector parallel to $\xi$ pointing in the same direction as $\xi$. Hence,

$$
\nabla_{x} \Phi_{\xi}^{g_{0}}=\nabla_{x} \beta_{\xi}^{g_{0}}=\xi
$$

for every $x \in \tilde{\Delta}$. 
Lemma 8.5 Let $g$ be a metric on $M$ with sys $(g) \geq 1$. Then the map $\Phi^{g}: \widetilde{M} \rightarrow \mathbb{R}^{\infty}$ induced by $g$ is 1-Lipschitz and admissible.

Proof Let $\xi \in \mathcal{S}_{0}$. Since $\operatorname{sys}_{g}\left(\alpha_{\xi}\right) \geq 1$, the $\xi$-coordinate maps $\Phi_{\xi}^{g}$ are 1-Lipschitz as an average of 1-Lipschitz functions. In particular, the condition (1) of Definition 3.4 is satisfied by the Rademacher theorem. Furthermore, the map $\Phi^{g}: \widetilde{M} \rightarrow \mathbb{R}^{\infty}$ is also 1-Lipschitz; cf Definition 7.1.

Let $x \in \widetilde{M}$ and $\gamma \in \Gamma$. Since $c_{\gamma . \xi}=\gamma . c_{\xi}$ and $d_{\widetilde{g}}$ is $\Gamma$-invariant, we have

$$
d_{\widetilde{g}}\left(\gamma \cdot x, c_{\gamma \cdot \xi}(t)\right)=d_{\tilde{g}}\left(x, c_{\xi}(t)\right) .
$$

We also have sys ${ }_{g}\left(\alpha_{\gamma . \xi}\right)=\operatorname{sys}_{g}\left(\alpha_{\xi}\right)$ since $\alpha_{\gamma . \xi}$ and $\alpha_{\xi}$ are conjugate in $\Gamma$. Thus,

$$
\begin{aligned}
& \beta_{\gamma \cdot \xi}^{g}(\gamma \cdot x)=\beta_{\xi}^{g}(x), \\
& \phi_{\gamma \cdot \xi}^{g}(\gamma \cdot x)=\phi_{\xi}^{g}(x) .
\end{aligned}
$$

Combined with the relations $(\gamma \cdot \xi)(i)=c_{\gamma \cdot \xi}^{\prime}(i / n)=\gamma \cdot c_{\xi}^{\prime}(i / n)=\gamma \cdot \xi(i)$ and the definition of $\Phi_{\xi}^{g}(\mathrm{cf}(8-7))$, we obtain

$$
\Phi_{s \gamma \cdot \xi}^{g}(\gamma \cdot x)=\Phi_{\xi}^{g}(x)
$$

Hence the equivariance of $d \Phi_{\xi}^{g}$; cf Definition 3.4 (3).

By definition of $\alpha_{\xi}$ (cf Definition 3.1), we have $\alpha_{\xi} \cdot c_{ \pm \xi}(t)=c_{ \pm \xi}(t \pm 1)$. Recall also that $c_{-\xi}(t)=c_{\xi}(-t), \alpha_{-\xi}=\alpha_{\xi}^{-1}$ and $\operatorname{sys}_{g}\left(\alpha_{-\xi}\right)=\operatorname{sys}_{g}\left(\alpha_{\xi}\right)$. Thus, the Busemann functions $\beta_{ \pm \xi}^{g}$ satisfy

$$
\begin{aligned}
\beta_{ \pm \xi}^{g}\left(\alpha_{\xi} \cdot x\right) & =\limsup _{t \rightarrow \infty} d_{\widetilde{g}}\left(\alpha_{\xi} \cdot x, c_{ \pm \xi}(t)\right)-t \operatorname{sys}_{g}\left(\alpha_{ \pm \xi}\right) \\
& =\limsup _{t \rightarrow \infty} d_{\widetilde{g}}\left(x, c_{ \pm \xi}(t \mp 1)\right)-(t \mp 1) \operatorname{sys}_{g}\left(\alpha_{ \pm \xi}\right) \mp \operatorname{sys}_{g}\left(\alpha_{\xi}\right) \\
& =\beta_{ \pm \xi}^{g}(x)-\operatorname{sys}_{g}\left(\alpha_{\xi}\right) .
\end{aligned}
$$

Hence

$$
\phi_{\xi}^{g}\left(\alpha_{\xi} \cdot x\right)=\phi_{\xi}^{g}(x)-\operatorname{sys}_{g}\left(\alpha_{\xi}\right) .
$$

Combined with the relation $\alpha_{\xi}=\alpha_{\xi(i)}$ and the definition of $\Phi_{\xi}$ (cf (8-7)), we obtain

$$
\Phi_{\xi}^{g}\left(\alpha_{\xi} \cdot x\right)=\Phi_{\xi}^{g}(x)-1
$$

Hence the condition (4) of Definition 3.4.

Now, from Equations (8-8) and (8-9), we derive

$$
\phi_{\alpha_{\xi}, \xi}^{g}(x)=\phi_{\xi}^{g}(x)+\operatorname{sys}_{g}\left(\alpha_{\xi}\right) .
$$


Let $\xi$ and $\xi^{\prime}=\xi(1)$ be two neighbor systolic directions pointing in the same direction. From the Equation (8-10) and the relations $\xi^{\prime}(i)=\xi(i+1)$ and $\xi(n)=\alpha_{\xi} \cdot \xi$, we deduce

$$
\begin{aligned}
\Phi_{\xi^{\prime}}^{g}(x) & =\left(\frac{1}{n} \sum_{i=0}^{n-1} \phi_{\xi(i+1)}^{g}(x)\right) / \operatorname{sys}_{g}\left(\alpha_{\xi}\right) \\
& =\left(\frac{1}{n} \sum_{j=1}^{n} \phi_{\xi(j)}^{g}(x)\right) / \operatorname{sys}_{g}\left(\alpha_{\xi}\right) \\
& =\left(\frac{1}{n} \sum_{j=0}^{n-1} \phi_{\xi(j)}^{g}(x)\right) / \operatorname{sys}_{g}\left(\alpha_{\xi}\right)+\frac{1}{n} \\
& =\Phi_{\xi}^{g}(x)+\frac{1}{n} .
\end{aligned}
$$

Repeatedly using this relation, we derive

$$
\Phi_{\xi^{\prime}}^{g}(x)=\Phi_{\xi}^{g}(x)+\frac{i}{n}
$$

for every pair of systolic directions $\xi$ and $\xi^{\prime}$ pointing in the same direction with $\xi^{\prime}=\xi(i)$ and $i \in \mathbb{Z}$.

The condition (2) of Definition 3.4 immediately follows.

\section{Induced metrics and two-forms of model admissible maps}

Let $\Phi^{0}$ be the 1-Lipschitz admissible map induced by the metric $g_{0}$ on $M_{0}$; see Definition 8.3.

Lemma 9.1 We have

$$
g_{\Phi^{0}}=\frac{3}{2} g_{0}
$$

Proof Let $x \in \widetilde{M}$ and $u \in T_{x} \widetilde{M}$. Denote by $\widetilde{\Delta}$ the triangle of $\mathcal{T}$ such that $x \in \widetilde{\Delta}$. Let $\left(\xi_{i}\right)_{i=1}^{3}$ be the three systolic directions with basepoints in $\widetilde{\Delta}$; cf Definition 3.3. We will write $\Phi_{i}^{0}=\Phi_{\xi_{i}}^{g_{0}}$.

$$
\begin{aligned}
\tilde{g}_{\Phi^{0}}(u, u) & =\left(\pi_{\tilde{\Delta}} \circ \Phi^{0}\right)^{*}\left(g_{E}\right)(u, u) \\
& =\sum_{i=1}^{3}\left|d_{x} \Phi_{i}^{0}(u)\right|^{2} .
\end{aligned}
$$


Suppose $u$ is the gradient of $\Phi_{j}^{0}$ at $x$ for the metric $\tilde{g}_{0}$, ie $u=\nabla_{x} \Phi_{j}^{0}$. Since the $\widetilde{g}_{0}$-dot product between $\nabla_{x} \Phi_{i}^{0}$ and $\nabla_{x} \Phi_{j}^{0}$ is equal to $1-\frac{3}{2} \delta_{i, j}$ (ie 1 when $i=j$ and $-\frac{1}{2}$ when $i \neq j$ ), we have

$$
\begin{aligned}
\tilde{g}_{\Phi^{0}}(u, u) & =\sum_{i=1}^{3} g_{0}\left(\nabla_{x} \Phi_{i}^{0}, \nabla_{x} \Phi_{j}^{0}\right)^{2} \\
& =\left\|\left(1,-\frac{1}{2},-\frac{1}{2}\right)\right\|_{E}^{2} \\
& =\frac{3}{2} \widetilde{g}_{0}(u, u) .
\end{aligned}
$$

since $u=\nabla \Phi_{j}^{0}$ is a $\widetilde{g}_{0}$-unit vector. We derive that

$$
\tilde{g}_{\Phi^{0}}=\frac{3}{2} \tilde{g}_{0}
$$

on $\widetilde{M}_{0}$ since the vectors $\nabla_{x} \Phi_{j}^{0}$ span the vector space $T_{x} \widetilde{M}$. Hence the result.

Lemma 9.2 We have

$$
\omega_{\Phi^{0}}=\frac{3 \sqrt{3}}{2} d g_{0}
$$

Proof We will use the same notation as in the proof of the previous lemma. By definition of $\widetilde{\omega}_{\Phi^{0}}$, we have (cf (6-1))

$$
\widetilde{\omega}_{\Phi^{0}}=\sum_{i=1}^{3} \operatorname{det}_{\widetilde{g}_{0}}\left(\nabla_{x} \Phi_{i}^{0}, \nabla_{x} \Phi_{i+1}^{0}\right) d \widetilde{g}_{0} .
$$

Now, the angle between the unit vectors $\nabla_{x} \Phi_{i}^{0}$ and $\nabla_{x} \Phi_{i+1}^{0}$ is equal to $2 \pi / 3$. Therefore,

Hence

$$
\begin{array}{r}
\operatorname{det}_{\tilde{g}_{0}}\left(\nabla_{x} \Phi_{i}^{0}, \nabla_{x} \Phi_{i+1}^{0}\right)=\frac{\sqrt{3}}{2} . \\
\tilde{\omega}_{\Phi^{0}}=\frac{3 \sqrt{3}}{2} d \widetilde{g}_{0} .
\end{array}
$$

\section{The $\mathcal{A}$-functional of an admissible map}

Definition 10.1 The $\mathcal{A}$-functional of an admissible map $\Phi: \widetilde{M} \rightarrow \mathbb{R}^{\infty}$ is defined as

$$
\mathcal{A}(\Phi)=\int_{M} \omega_{\Phi}
$$


Let $g$ be a metric on $M$ with sys $(g) \geq 1$. Consider the admissible map $\Phi^{g}$ induced by $g$. Define $\widetilde{\varphi}^{g}: \widetilde{M} \rightarrow \mathbb{R}^{\infty}$ as the difference $\widetilde{\varphi}^{g}=\Phi^{g}-\Phi^{0}$, where $\Phi^{0}$ is the admissible map induced by the metric $g_{0}$ on $M_{0}$.

Lemma 10.2 Let $\xi \in \mathcal{S}_{0}$. The function $\widetilde{\varphi}_{\xi}^{g}: \widetilde{M} \rightarrow \mathbb{R}$ passes to the quotient by $\left\langle\alpha_{\xi}\right\rangle$ and induces a function denoted by $\varphi_{\xi}^{g}: \widetilde{M} /\left\langle\alpha_{\xi}\right\rangle \rightarrow \mathbb{R}$.

Proof Passing to the quotient by $\left\langle\alpha_{\xi}\right\rangle$, the real-valued function $\Phi_{\xi}^{g}$ induces a map $\overline{\Phi_{\xi}^{g}}: \widetilde{M} /\left\langle\alpha_{\xi}\right\rangle \rightarrow \mathbb{R} / \mathbb{Z}$ which takes a (simple) systolic loop of $\widetilde{M} /\left\langle\alpha_{\xi}\right\rangle$ to a generator of the fundamental group of $\mathbb{R} / \mathbb{Z}$. This map $\overline{\Phi_{\xi}^{g}}$ induces an isomorphism in homotopy between $\pi_{1}\left(\widetilde{M} /\left\langle\alpha_{\xi}\right\rangle\right)$ and $\pi_{1}(\mathbb{R} / \mathbb{Z})$. Therefore, the map $\varphi_{\xi}^{g}$ passes to the quotient and induces a map $\frac{\varphi_{\xi}^{g}}{\tilde{g}}: \widetilde{M} /\left\langle\alpha_{\xi}\right\rangle \rightarrow \mathbb{R} / \mathbb{Z}$ which is homotopically trivial and lifts to the desired function $\varphi_{\xi}^{g}: \widetilde{M} /\left\langle\alpha_{\xi}\right\rangle \rightarrow \mathbb{R}$.

Definition 10.3 Let $\xi \in \mathcal{S}_{0}$ be a systolic direction of $\widetilde{M}_{0}$. Recall that the systolic band $\widetilde{B}_{\xi}$ of $M$ induced by $\xi$ is the union of all the triangles of $\mathcal{T}$ through which $c_{\xi}$ passes. The projection $B_{\xi}$ of $\widetilde{B}_{\xi}$ to $M_{0}$, which agrees with the quotient of $\widetilde{B}_{\xi}$ by $\left\langle\alpha_{\xi}\right\rangle$, is also called a systolic band of $M_{0}$.

We assign to every triangle $\Delta \in \mathcal{T}$ lying in a systolic band $B$ the systolic direction $\xi_{\Delta, B}$ with basepoint in $\Delta$ pointing in the same direction as the edge of $\Delta$ lying in the boundary of $B$ (the edges of a triangle inherit the orientation of the triangle).

Let $\mathcal{D}$ be a collection of triangles of $\mathcal{T}$ whose union forms a fundamental domain of $M$ in $\widetilde{M}$ as in Figures 1 and 2. By definition of $\mathcal{A}$, we have

$$
\begin{aligned}
\mathcal{A}\left(\Phi^{g}\right)-\mathcal{A}\left(\Phi^{0}\right) & =\sum_{\tilde{\Delta} \in \mathcal{D}} \int_{\widetilde{\Delta}} \sum_{i=1}^{3} d \widetilde{\varphi}_{i}^{g} \wedge d \Phi_{i+1}^{0}+d \Phi_{i}^{0} \wedge d \widetilde{\varphi}_{i+1}^{g}+d \widetilde{\varphi}_{i}^{g} \wedge d \widetilde{\varphi}_{i+1}^{g} \\
& =\sum_{\tilde{\Delta} \in \mathcal{D}} \int_{\widetilde{\Delta}} \sum_{i=1}^{3} d \widetilde{\varphi}_{i}^{g} \wedge d \Phi_{i+1}^{0}+d \Phi_{i}^{0} \wedge d \widetilde{\varphi}_{i+1}^{g}
\end{aligned}
$$

where

$$
\Phi_{i}^{g}=\Phi_{\xi_{\widetilde{\Delta}, i}}^{g}, \quad \Phi_{i}^{0}=\Phi_{\xi_{\widetilde{\Delta}, i}}^{0} \quad \text { and } \quad \tilde{\varphi}_{i}^{g}=\tilde{\varphi}_{\xi_{\widetilde{\Delta}, i}}^{g} .
$$

Here, the $\xi_{\widetilde{\Delta}, i}$ 's, with $i \in\{1,2,3\}$, are the three systolic directions arising from the triangle $\widetilde{\Delta}$ of $\mathcal{D}$. 
The following proposition amounts to the criticality of the $\mathcal{A}$-functional at $\Phi^{0}$.

Proposition 10.4 We have

$$
I_{g}:=\sum_{\tilde{\Delta} \in \mathcal{D}} \int_{\widetilde{\Delta}} \sum_{i=1}^{3} d \widetilde{\varphi}_{i}^{g} \wedge d \Phi_{i+1}^{0}+d \Phi_{i}^{0} \wedge d \widetilde{\varphi}_{i+1}^{g}=0 .
$$

Proof By rearranging the terms of the sum, we can write $I_{g}$ as

$$
I_{g}=\sum_{\widetilde{\Delta} \in \mathcal{D}} \sum_{i=1}^{3} \int_{\widetilde{\Delta}} d \tilde{\varphi}_{i}^{g} \wedge\left(d \Phi_{i+1}^{0}-d \Phi_{i-1}^{0}\right) .
$$

Now, note that $d \Phi_{i+1}^{0}-d \Phi_{i-1}^{0}=(\sqrt{3} / 2)\left(d \Phi_{i}^{0}\right)^{\star}$, where $\left(d \Phi_{i}^{0}\right)^{\star}$ is the image of $d \Phi_{i}^{0}$ by the Hodge operator on the one-form space of $\widetilde{M}_{0}$. Thus,

$$
I_{g}=\frac{\sqrt{3}}{2} \sum_{\widetilde{\Delta} \in \mathcal{D}} \sum_{i=1}^{3} \int_{\tilde{\Delta}} d \tilde{\varphi}_{i}^{g} \wedge\left(d \Phi_{i}^{0}\right)^{\star} .
$$

The one-forms $\left(d \Phi_{i}^{0}\right)^{\star}$ are constant on the systolic bands $\widetilde{B}_{\xi}$ of $\widetilde{M}_{0}$. We will still denote by $\left(d \Phi_{i}^{0}\right)^{\star}$ the one-form obtained by passing to the quotient on $B_{\xi}$. By rearranging the terms of the sum and passing to the quotient, we can write (10-2) as

$$
I_{g}=\frac{\sqrt{3}}{2} \sum_{B} \sum_{\Delta \subset B} \int_{\Delta} d \varphi_{\Delta, B}^{g} \wedge\left(d \Phi_{\Delta, B}^{0}\right)^{\star}
$$

where the first sum is taken over all the systolic bands $B$ of $M_{0}$ and the second over all the triangles $\Delta$ of $\mathcal{T}$ lying in $B$. Here, $\varphi_{\Delta, B}^{g}=\varphi_{\xi_{\Delta, B}}^{g}$ and $\Phi_{\Delta, B}^{g}=\Phi_{\xi_{\Delta, B}}^{g}$ where $\xi_{\Delta, B}$ is defined in Definition 3.2.

Now, fix a triangle $\Delta_{0}$ of $\mathcal{T}$ lying in $B$. Set $\varphi_{B}^{g}=\varphi_{\Delta_{0}, B}^{g}$ and $\nu_{B}^{0}=\left(d \Phi_{\Delta_{0}, B}^{0}\right)^{\star}$. Let $\Delta$ be another triangle of $\mathcal{T}$ lying in $B$. If $\Delta$ and $\Delta_{0}$ have an edge lying in the same connected component of $\partial B$, then $d \varphi_{\Delta, B}^{g}=d \varphi_{B}^{g}$ from Definition 3.4 (2), and $\left(d \Phi_{\Delta, B}^{0}\right)^{\star}=v_{B}^{0}$. Similarly, if $\Delta$ and $\Delta_{0}$ do not have any edge lying in the same connected component of $\partial B$, then $d \varphi_{\Delta, B}^{g}=-d \varphi_{B}^{g}$ and $\left(d \Phi_{\Delta, B}^{0}\right)^{\star}=-v_{B}^{0}$. In both cases,

Therefore,

$$
\begin{gathered}
d \varphi_{\Delta, B}^{g} \wedge\left(d \Phi_{\Delta, B}^{0}\right)^{\star}=d \varphi_{B}^{g} \wedge v_{B}^{0} . \\
I_{g}=\frac{\sqrt{3}}{2} \sum_{B} \int_{B} d \varphi_{B}^{g} \wedge v_{B}^{0} .
\end{gathered}
$$


Since the one-form $v_{B}^{0}$ is constant on $B$, and therefore closed on $B$, we deduce from Stokes' formula that

$$
I_{g}=\frac{\sqrt{3}}{2} \sum_{B} \int_{\partial B} \varphi_{B}^{g} v_{B}^{0} .
$$

But the one-form $v_{B}^{0}$ vanishes on the vectors tangent to $\partial B$. Thus, $I_{g}$ equals zero.

\section{Proof of the main result}

Let $\Phi=\Phi^{g}$ be the 1 -Lipschitz admissible map induced by a metric $g$ on $M$. From Lemma 9.2, we derive that

$$
\mathcal{A}\left(\Phi^{0}\right)=\frac{3 \sqrt{3}}{2} \operatorname{area}\left(g_{0}\right) .
$$

Now, from Corollary 7.3 and Lemma 6.1, we have

$$
\mathcal{A}\left(\Phi^{g}\right) \leq \sqrt{3} \operatorname{area}\left(g_{\Phi}\right) \leq \frac{3 \sqrt{3}}{2} \operatorname{area}(g) .
$$

Let $\left(g_{t}\right)$ be a slow metric variation of $g_{0}$ with $\operatorname{sys}\left(g_{t}\right)=1$. By definition of a slow variation, we deduce from the Cauchy-Schwarz inequality that the term $J_{g_{t}}$ in (10-1) is negligible with respect to $t$. Thus, Proposition 10.4 implies that

Hence,

$$
\mathcal{A}\left(\Phi^{g_{t}}\right)-\mathcal{A}\left(\Phi^{0}\right)=o(t) .
$$

$$
\operatorname{area}\left(g_{t}\right) \geq \operatorname{area}\left(g_{0}\right)+o(t) \text {. }
$$

\section{Examples of slow metric variations}

The goal of this section is to present examples of slow metric variations and, more generally, to show how one can prove that a metric variation is slow.

\subsection{A general bound for not-necessarily slow metric variations}

Let $\left(g_{t}\right)_{t \geq 0}$ be a smooth deformation of $g_{0}$. More precisely, $g_{t}$ is a Riemannian metric with conical singularities on $M$ smoothly varying with $t$ on $M_{0} \backslash$ \{conical singularities\} (as a smooth section of the bundle of symmetric 2-forms on $M_{0} \backslash$ \{conical singularities\}). Recall from [27] that a point $p$ of $M$ is a conical singularity of order $\tau$ (or of angle $\theta=2 \pi(\tau+1))$ of the metric $g$ if there exists a nonsingular conformal map $z: U \rightarrow \mathbb{C}$ defined in a neighborhood $U$ of $p$ such that $z(p)=0$ and $g=f(z)|z|^{2 \tau}|d z|^{2}$ in $U$, for some continuous positive function $f$. 
From [13], if $p$ and $q$ are two nonconjugate points on a complete simply connected manifold endowed with a Riemannian metric $\widetilde{g}$, there exists a neighborhood of $\widetilde{g}$ such that for every metric $\tilde{h}$ in this neighborhood, the points $p$ and $q$ are connected by a unique $\tilde{h}$-geodesic arc smoothly depending on $\tilde{h}$. Thus, the $g_{t}$-minimizing arcs joining pairs of adjacent singularities smoothly vary with $t$, for $t$ small enough. Therefore, by taking the pullback of $g_{t}$ under a one-parameter family of diffeomorphisms converging to the identity map when $t$ goes to zero, we can assume that the edges of the triangulation $\mathcal{T}$ are $g_{t}$-minimizing arcs for $t$ small enough.

Recall that the systolic band $\widetilde{B}_{\xi}$ induced by a systolic direction $\xi \in \mathcal{S}_{0}$ (cf Definition 3.2) is a flat strip in $\widetilde{M}_{0}$ composed of equilateral flat triangles of $\mathcal{T}$. The trajectories (for positive time) of the dynamical system generated by $-\nabla_{g_{0}} \beta_{\xi}^{g_{0}}$ (where $\beta_{\xi}^{g_{0}}$ is the Busemann function defined by $\xi$; cf Definition 8.2) arising from the points of $\widetilde{B}_{\xi}$ are semilines parallel to the boundary of the flat strip $\widetilde{B}_{\xi}$ and pointing in the same direction as $c_{\xi}$; cf Definition 3.1. If the metric has nonpositive curvature (the case we will focus on), the Busemann function $\beta_{\xi}^{g_{t}}$ is differentiable and the trajectory $\gamma_{t}^{x}$ of $-\nabla_{g_{t}} \beta_{\xi}^{g_{t}}$ arising from $x$ is complete and leaves every compact set.

The following result shows that for $t$ small enough the trajectories of $-\nabla_{g_{t}} \beta_{\xi}^{g_{t}}$ are still trapped in $\widetilde{B}_{\xi}$.

Lemma 12.1 For $t$ small enough, every complete minimizing $\tilde{g}_{t}$-geodesic trajectory $\gamma_{t}$ leaving $\widetilde{B}_{\xi}$ does not stay at bounded distance from $c_{\xi}$.

In particular, for $t$ small enough, the trajectories of $-\nabla_{g_{t}} \beta_{\xi}^{g t}$ arising from the points of $\widetilde{B}_{\xi}$ lie in $\widetilde{B}_{\xi}$.

Proof We will assume that all the geodesics are parametrized by their arclength. Let $\widetilde{B}_{\xi}^{+}$denote the $\widetilde{g}_{0}$-convex neighborhood of $\widetilde{B}_{\xi}$ formed of the triangles of $\mathcal{T}$ with a vertex in $\widetilde{B}_{\xi}$. For $t$ small enough, the boundary of $\widetilde{B}_{\xi}$ is made of $\widetilde{g}_{t}-$ minimizing segments and the angles of the singularities of $\tilde{g}_{t}$ are greater than $2 \pi$. Therefore, for $t$ small enough, the trajectories $\gamma_{t}$ leaving $\widetilde{B}_{\xi}$ also leave $\widetilde{B}_{\xi}^{+}$. By translating the trajectories if necessary, we can extract a sequence $\gamma_{t_{n}}$, where $t_{n} \rightarrow 0$, leaving $\widetilde{B}_{\xi}^{+}$ with a speed vector $u_{n}$ such that $\left(u_{n}\right)$ converges to a $\widetilde{g}_{0}$-unit vector $u_{\infty}$ pointing outside the interior of $\widetilde{B}_{\xi}^{+}$. Since the conical singularities of $\tilde{g}_{0}$ are greater than $2 \pi$, the complete minimizing $\widetilde{g}_{0}$-geodesic trajectories $\gamma_{\infty}$ generated by $u_{\infty}$ do not stay at bounded distance from $c_{\xi}$. (Note that the trajectories $\gamma_{\infty}$ are not necessarily uniquely determined by $u_{\infty}$ as they may pass through conical singularities.) On the other hand, there exists a trajectory $\gamma_{\infty}$ as above such that $\left(\gamma_{t_{n}}\right)$ converges (up to a subsequence) to $\gamma_{\infty}$ in restriction to any given bounded open set of $\widetilde{M}$. The conclusion follows. 
We can now prove a general bound on the variation of the gradient of Busemann functions with respect to any (not-necessarily slow) metric deformation.

Proposition 12.2 Let $\left(g_{t}\right)_{t \geq 0}$ be a smooth deformation of $g_{0}$ as above. Then for every systolic direction $\xi \in \mathcal{S}_{0}$ and every triangle $\widetilde{\Delta}$ of $\mathcal{T}$ lying in $\widetilde{B}_{\xi}$, we have

$$
\left\|d \beta_{\xi}^{g_{t}}-d \beta_{\xi}^{g_{0}}\right\|_{L^{\infty}(\tilde{\Delta})}=\mathcal{O}(\sqrt{t}) .
$$

Remark 12.3 We do not know whether this $\mathcal{O}(\sqrt{t})$-bound can be replaced with a $o(\sqrt{t})$-bound as in the definition of slow metric variations; cf Definition 1.1. A positive answer would show that any metric variation is slow.

Proof Fix $x \in \widetilde{\Delta}$ a point where $\beta_{\xi}^{g_{t}}$ is differentiable. Let $\mathfrak{h}$ be the $\widetilde{g}_{0}$-segment of $\widetilde{B}_{\xi}$ passing through $x$ and $\widetilde{g}_{0}$-orthogonal to the boundary components of $\widetilde{B}_{\xi}$. Set $x_{i}=\alpha_{\xi}^{i} \cdot x$ and $\mathfrak{h}_{i}=\alpha_{\xi}^{i} \cdot \mathfrak{h} ; \operatorname{cf}$ Definition 3.1. Denote by $h$ the distance function to $c_{\xi}$ with respect to $\tilde{g}_{0}$. Suppose for simplicity that the angle $\theta_{0}=\theta_{0}(x)$ between $-\nabla_{g_{0}} \beta_{\xi}^{g_{0}}\left(x_{0}\right)$ and $-\nabla_{g_{t}} \beta_{\xi}^{g_{t}}\left(x_{0}\right)$ is positive. Note that the function $\theta_{0}$ tends to zero in $L^{\infty}(\tilde{\Delta})$ when $t$ goes to zero. Since the metric deformation is smooth and $\operatorname{sys}_{g_{0}}\left(\alpha_{\xi}\right)=1$ (cf (8-1)), the trajectory $\gamma_{t}^{x}$ of $-\nabla_{g_{t}} \beta_{\xi}^{g_{t}}$ arising from $x_{0}$, which is geodesic for $\tilde{g}_{t}$, hits $\mathfrak{h}_{1}$ at a point $x_{1}$ such that $h\left(x_{1}\right)-h\left(x_{0}\right) \geq \sin \theta_{0}-C t$ for $t$ small enough, where, by compactness, $C$ is an absolute constant (ie, a constant depending only on $g_{0}$ and the derivatives of the metric family $\left(g_{t}\right)$ at $t=0$, but not on $t$ and $\left.x_{0}\right)$. Furthermore, the angle $\theta_{1}$ between $-\nabla_{g_{0}} \beta_{\xi}^{g_{0}}\left(x_{1}\right)$ and $-\nabla_{g_{t}} \beta_{\xi}^{g_{t}}\left(x_{1}\right)$ is greater or equal to $\theta_{0}-C^{\prime} t$, where $C^{\prime}$ is another absolute constant. We repeat this process again and again. As a result, we obtain a sequence of points $x_{i}$ along the trajectory $\gamma_{t}^{x}$ and a sequence of angles $\theta_{i}$ between $-\nabla_{g_{0}} \beta_{\xi}^{g_{0}}\left(x_{i}\right)$ and $-\nabla_{g_{t}} \beta_{\xi}^{g_{t}}\left(x_{i}\right)$ is such that

$$
\begin{aligned}
h\left(x_{i+1}\right)-h\left(x_{i}\right) & \geq \sin \theta_{i}-C t, \\
\theta_{i} & \geq \theta_{0}-i C^{\prime} t
\end{aligned}
$$

This last inequality implies that $\theta_{i}$ is positive for $i<i_{0}:=\left[\theta_{0} / C^{\prime} t\right]$. Note that for $t$ small enough, $\theta_{i}$ is less than $\pi / 4$ and so $\sin \theta_{i} \geq \frac{9}{10} \theta_{i}$. Thus, summing the inequality (12-1) from $i=0$ to $i_{0}-1$ and using (12-2), we derive that for $t$ small enough,

$$
\begin{aligned}
h\left(x_{i_{0}}\right)-h\left(x_{0}\right) \geq \sum_{i=0}^{i_{0}-1} \sin \theta_{i}-i_{0} C t & \geq \frac{9}{10} \sum_{i=0}^{i_{0}-1} \theta_{i}-\frac{C}{C^{\prime}} \theta_{0} \\
& \geq \frac{9}{10}\left(i_{0} \theta_{0}-\frac{i_{0}\left(i_{0}-1\right)}{2} C^{\prime} t\right)-\frac{C}{C^{\prime}} \theta_{0}
\end{aligned}
$$


Now, since $\gamma_{t}^{x}$ lies in $\widetilde{B}_{\xi}$ from Lemma 12.1 , the sequence $h\left(x_{i}\right)$ is uniformly bounded, for instance by 1 . Thus, from (12-3) and the expression of $i_{0}$, the angle $\theta_{0}$ satisfies some inequality

$$
\frac{a}{t} \theta_{0}^{2}-b \theta_{0}-1 \leq 0
$$

where $a$ and $b$ are positive absolute constants. We immediately deduce that

$$
\theta_{0} \leq \frac{b+\sqrt{b^{2}+4 a / t}}{2 a / t}=\mathcal{O}(\sqrt{t}) .
$$

Hence the result.

\subsection{Changing the edge lengths}

A piecewise flat Calabi surface $M_{0}=\left(M, g_{0}\right)$ is composed of Euclidean equilateral triangles with the same side length. We can deform the metric $g_{0}$ into another piecewise flat metric by changing the lengths of the sides of the Euclidean triangles of $\mathcal{T}$ tilling the surface; cf Section 2. This yields a 48-dimensional space of deformations for the Calabi surface modeled on Fermat's quartic and a 84-dimensional space of deformations for the one modeled on Klein's quartic. We consider a one-parameter family $\left(g_{t}\right)_{t \geq 0}$ of such deformations, where the lengths of the sides of the Euclidean triangles of $\mathcal{T}$ smoothly depend on $t$. We denote by $\mathcal{T}_{t}$ and $\mathcal{T}_{t}$ the deformations of the triangulations given by $\mathcal{T}$ and $\mathcal{T}$. For $t$ small enough, the conical singularities of the piecewise flat metric $g_{t}$ have nonpositive curvature in Alexandrov's sense and the Busemann functions of $g_{t}$ are differentiable everywhere off the conical singularities.

Proposition 12.4 Let $\widetilde{\Delta}$ be a triangle of $\mathcal{T}$ and $\xi \in \mathcal{S}_{0}$ be a systolic direction with basepoint in $\widetilde{\Delta}$. Then the metric family $\left(g_{t}\right)_{t \geq 0}$ satisfies

$$
\left\|\left(d \beta_{\xi}^{g_{t}}-d \beta_{-\xi}^{g_{t}}\right)-\left(d \beta_{\xi}^{g_{0}}-d \beta_{-\xi}^{g_{0}}\right)\right\|_{L^{2}(\tilde{\Delta})}=\mathcal{O}(t) .
$$

In particular, the one-parameter family of metrics $\left(g_{t}\right)_{t \geq 0}$ is a slow metric variation.

Remark 12.5 The estimate (12-4) is stronger than the estimate (1-1) in the definition of a slow metric variation; cf Definition 1.1. Indeed, it yields a $\mathcal{O}(t)$-bound, which is stronger than a $o(\sqrt{t})$-bound. This still leaves room for improvement and for describing new slow metric variations.

It will be convenient to introduce the following definition. 
Definition 12.6 We will write that $a(t) \lesssim b(t)$ if there exists a positive absolute constant $C$ (ie, a constant depending only on $g_{0}$ and the derivatives of the metric family $\left(g_{t}\right)$ at $t=0$, but not on $\left.t\right)$ such that $a(t) \lesssim C b(t)$ for $t$ small enough. Similarly, we define $a(t) \gtrsim b(t)$. If both $a(t) \leq b(t)$ and $a(t) \gtrsim b(t)$, we will write $a(t) \simeq b(t)$.

We will also use this definition for functions $a(x, t)$ depending on a variable $x$. In this case, we require the absolute constant $C$ to be independent of $x$.

Proof of Proposition 12.4 As recalled in Section 12.1, the systolic band $\widetilde{B}_{\xi}$ of $M_{0}$ induced by $\xi$ is a flat strip in $\widetilde{M}_{0}$ composed of equilateral flat triangles of $\mathcal{T}$. This band can also be seen as a horizontal strip in $\mathbb{R}^{2}$ endowed with the Euclidean metric. Moving along $c_{\xi}$ in the positive direction, we denote by $\widetilde{\Delta}_{1}$ the triangle of $\mathcal{T}$ in $\widetilde{B}_{\xi}$ adjacent to $\widetilde{\Delta}_{0}=\widetilde{\Delta}$, by $\widetilde{\Delta}_{2}$ the triangle of $\mathcal{T}$ in $\widetilde{B}_{\xi}$ adjacent to $\widetilde{\Delta}_{1}$, and so on. Similarly, we define $\widetilde{\Delta}_{k}$ for $k$ negative by moving along $c_{\xi}$ in the negative direction.

As $t$ varies, the triangles of $\mathcal{T}_{t}$ and $\mathcal{T}_{t}$ are smoothly deformed. In order to compare the geometry of $g_{0}$ and $g_{t}$, it will be convenient to consider the triangles $\widetilde{\Delta}_{k}$ and their smooth deformations $\widetilde{\Delta}_{k}^{t}$ embedded in $\mathbb{R}^{2}$; see Figure 3. (This will implicitly be used in the sequel.) This can be done by keeping both the center of $\widetilde{\Delta}_{0}^{t}$ and the direction of a given edge of $\tilde{\Delta}_{0}^{t}$ fixed in $\mathbb{R}^{2}$. With this identification, the restriction of $g_{t}$ to the union $\widetilde{B}_{\xi}^{t}$ of the $\widetilde{\Delta}_{k}^{t}$ agrees with (more precisely, is the pullback of) the Euclidean metric of the plane. Note that even for small $t$, the center of $\tilde{\Delta}_{k}^{t}$ can be far from the center of $\widetilde{\Delta}_{k}$ (of course, this occurs only for $k$ large enough) and the band $\widetilde{B}_{\xi}^{t}$ may no longer be embedded in $\mathbb{R}^{2}$. However, we will later see that large portions of the band $\widetilde{B}_{\xi}^{t}$ do not overlap in $\mathbb{R}^{2}$.

A geodesic loop of a piecewise flat surface with conical singularities which does not pass through a singularity can be translated without increasing its length until it reaches a singularity. Thus, every homotopy class can be represented by a shortest representative passing through a conical singularity. In particular, the lift of some $\xi$-systolic loop of $\left(M, g_{t}\right)$ passes through some vertex (conical singularity) $x_{0}$ of $\tilde{B}_{\xi}^{t}$ at distance $\lesssim 1$ from $\widetilde{\Delta}$. Here, a $\xi$-systolic loop of $M$ is the projection of a systolic loop of $\widetilde{M} /\left\langle\alpha_{\xi}\right\rangle$ on $M$. Consider now the piecewise straight line $c$ of $\mathbb{R}^{2}$ composed of the segments $c_{i}$ of $\mathbb{R}^{2}$ with endpoints $x_{i}=\alpha_{\xi}^{i} \cdot x_{0}$ and $x_{i+1}$ in $\widetilde{B}_{\xi}^{t}$. Let $v$ be the angle between the directions of $c_{i}$ and $c_{i+1}$ (it does not depend on $i$ since $\alpha_{\xi}$ acts by isometry on $\widetilde{B}_{\xi}^{t}$ ). Since the triangles $\widetilde{\Delta}_{k}^{t}$ smoothly vary in $\mathbb{R}^{2}$, we have $|v| \lesssim t$.

Suppose that $v$ is different from zero. Then $c$ lies outside the disk of $\mathbb{R}^{2}$ bounded by the circle $\mathcal{C}_{\rho}$ of radius $\rho=\ell /(2 \tan (v / 2)) \simeq 1 / t$ passing through the midpoints of the segments $c_{i}$, where $\ell$ is the length of $c_{i}$; see Figure 3. Changing the origin $O$ of $\mathbb{R}^{2}$ if necessary, we can assume that the disk is centered at $O$. Since the boundary 


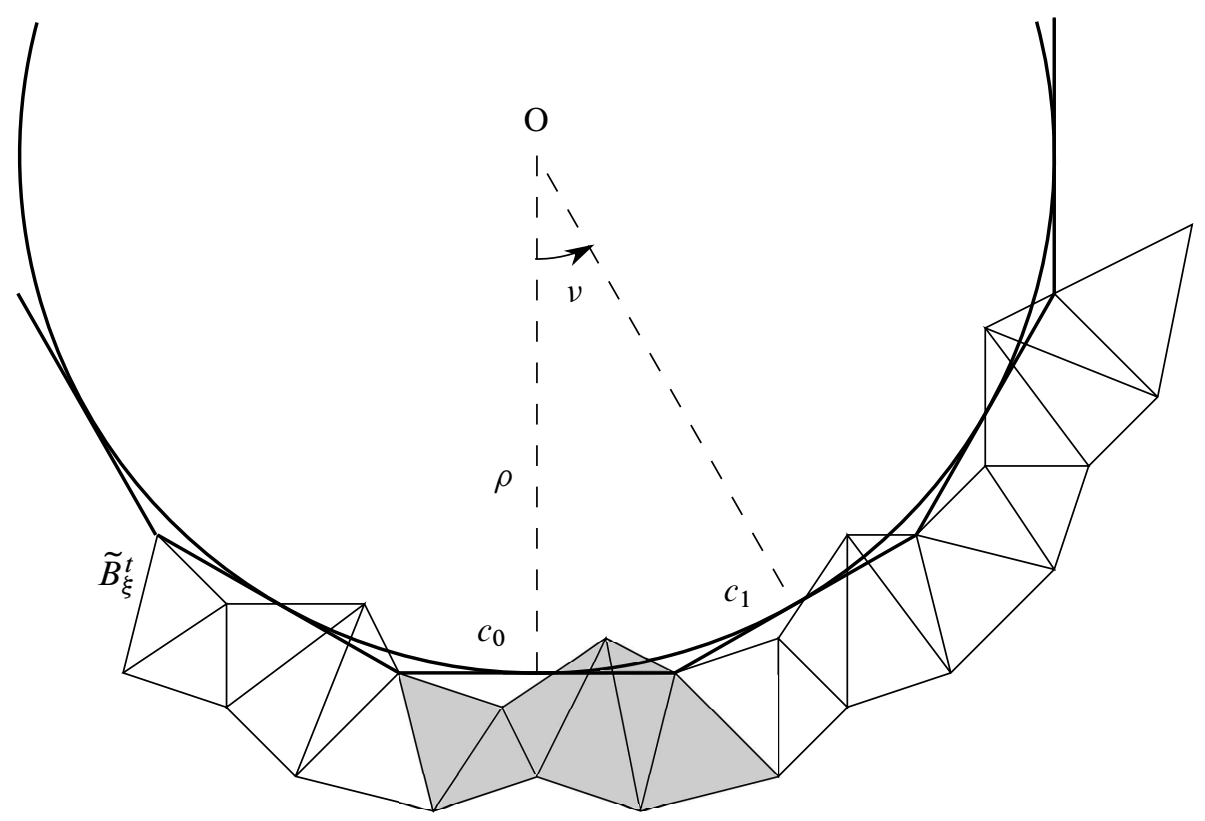

Figure 3: In gray, a fundamental domain for the projection of $\widetilde{B}_{\xi}^{t}$

component $\partial_{0} \widetilde{B}_{\xi}^{t}$ of $\widetilde{B}_{\xi}^{t}$ passing through $x_{0}$ is at Hausdorff distance $\lesssim t$ from $c$ in $\mathbb{R}^{2}$, it lies in a tubular neighborhood of $\mathcal{C}_{\rho}$ of width $\simeq t$, that is, an annulus $A(r, R)$ of $\mathbb{R}^{2}$ centered at $O$ of inner radius $r$ and outer radius $R$, where $\rho-r \simeq t$ and $R-\rho \simeq t$; see Figure 4. In particular, the band $\widetilde{B}_{\xi}^{t}$ is bounded in $\mathbb{R}^{2}$.

The area of $\tilde{\Delta} \cap D(R+t)$ is $\lesssim t$, where $D(R+t)$ is the disk of radius $R+t$ centered at $O$. Thus, from Proposition 12.2,

$$
\left\|d \beta_{\xi}^{g_{t}}-d \beta_{\xi}^{g_{0}}\right\|_{L^{2}(\tilde{\Delta} \cap D(R+t))} \lesssim t \sqrt{t} .
$$

Let us now bound the $L^{\infty}$-variations of $d \beta_{\xi}^{g_{s}}-d \beta_{-\xi}^{g_{s}}$ between $s=0$ and $s=t$ on $\widetilde{\Delta} \backslash D(R+t)$. As in Lemma 12.1, for $t$ small enough, every geodesic of $\left(\widetilde{M}, \widetilde{g}_{t}\right)$ leaving $\widetilde{B}_{\xi}^{t}$ diverges so fast that it does not stay at bounded distance from $c_{\xi}$. (This is due to the angles of the singularities of $g_{t}$ which are greater than $2 \pi$.) Thus, for $t$ small enough, the trajectories of $-\nabla_{g_{t}} \beta_{\xi}^{g_{t}}$ arising from the points of $\widetilde{B}_{\xi}^{t} \backslash \underset{\widetilde{B}^{t}}{D}(R+t)$ (which are geodesics possibly passing through conical singularities) lie in $\tilde{B}_{\xi}^{t}$. Since they are length-minimizing geodesics, each of these trajectories lies in the convex cone bounded by two semilines arising from the same starting point as the trajectory and pointing in the same direction as $\xi$, where the first semiline is tangent to $\mathcal{C}_{r}$ and the second is tangent to $\mathcal{C}_{R}$; see Figure 4. Thus, for every $x$ in $\widetilde{\Delta} \backslash D(R+t)$, the vector $-\nabla_{g_{t}} \beta_{\xi}^{g}(x)$ points into the convex cone previously described with vertex $x$. 
Using a simple trigonometric argument, we deduce that

$$
\frac{r}{R+d} \leq \cos \theta \leq \frac{R}{R+d}
$$

where $\theta=\theta(x)$ is the angle between $u(x)$ and $-\nabla_{g_{t}} \beta_{\xi}^{g_{t}}(x)$ (here, $u(x)$ is the vector based at $x$ orthogonal to the line $O x$ and pointing in the same direction as $\xi$ ) and $d$ is the distance from $x$ to the disk bounded by $\mathcal{C}_{R}$. Strictly speaking, we need to estimate the angle in $\mathbb{R}^{2}$ between $-\nabla_{g_{0}} \beta_{\xi}^{g_{0}}(x)$ and $-\nabla_{g_{t}} \beta_{\xi}^{g_{t}}(x)$, but since this angle agrees with $\theta$ up to some constant $\lesssim t$, we can work with $\theta$ instead.

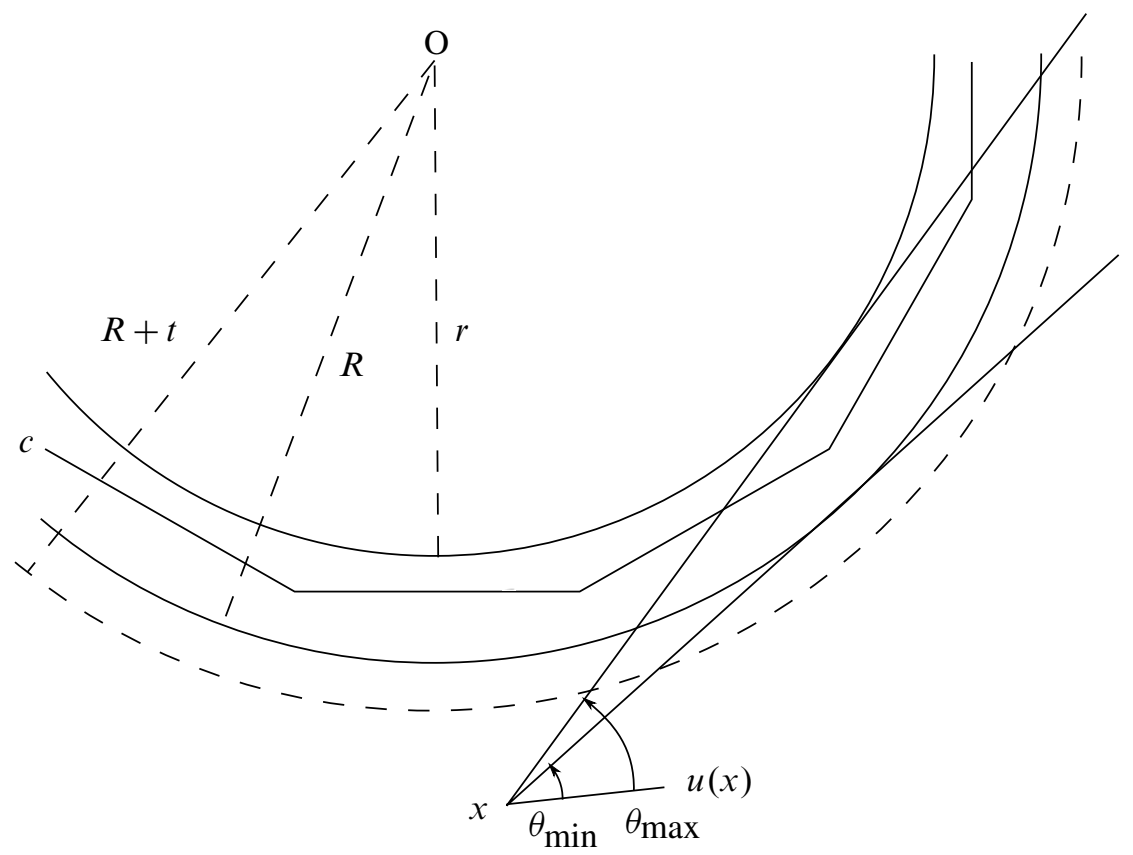

Figure 4

As $x$ lies in $\tilde{\Delta} \backslash D(R+t)$, we have $t \leq d \lesssim 1$. Combined with the estimates on $r$ and $R$, namely $r \simeq R \simeq 1 / t$ and $r-R \simeq t$, we derive from (12-5) that $t \lesssim \theta \lesssim \sqrt{t}$. This does not yield the desired $o(\sqrt{t})$-bound on $\theta$, only a $\mathcal{O}(\sqrt{t})$-bound. To obtain a $o(\sqrt{t})$-bound, we have to use a symmetry argument and deal with $-\xi$ as well.

Set $\theta_{+}=\theta$. Replacing $\xi$ with $-\xi$, we define $\theta_{-}$as the angle between $-u(x)$ (which almost agrees with $\left.-\nabla_{g_{0}} \beta_{-\xi}^{g_{0}}(x)\right)$ and $-\nabla_{g_{t}} \beta_{-\xi}^{g_{t}}(x)$. The angle $\theta_{-}$satisfies the same relation $t \lesssim \theta_{-} \lesssim \sqrt{t}$ and the same bound (12-5) as $\theta_{+}$. Therefore, since $|R-r| \lesssim t$, we deduce that

$$
\left|\cos \theta_{+}-\cos \theta_{-}\right| \leq \frac{|R-r|}{R+d} \lesssim t^{2} .
$$


From the mean value theorem, there exists $\theta_{c}$ between $\theta_{+}$and $\theta_{-}$(and so $\theta_{c} \gtrsim t$ ) such that

$$
\begin{aligned}
\left|\cos \theta_{+}-\cos \theta_{-}\right| & =\left|\sin \theta_{c}\right| \cdot\left|\theta_{+}-\theta_{-}\right| \\
& \gtrsim t \cdot\left|\theta_{+}-\theta_{-}\right| .
\end{aligned}
$$

Hence, $\left|\theta_{+}-\theta_{-}\right| \lesssim t$. Therefore,

$$
\begin{aligned}
\left|\left(d \beta_{\xi}^{g_{t}}-d \beta_{-\xi}^{g_{t}}\right)-\left(d \beta_{\xi}^{g_{0}}-d \beta_{-\xi}^{g_{0}}\right)\right| & \simeq\left|\cos \theta_{+}+\cos \theta_{-}-2+i\left(\sin \theta_{+}-\sin \theta_{-}\right)\right| \\
& \lesssim t
\end{aligned}
$$

on $\tilde{\Delta} \backslash D(R+t)$.

Suppose now that $v$ is equal to zero. Then $c$ is a straight line in $\mathbb{R}^{2}$. Since the boundary component $\partial_{0} \widetilde{B}_{\xi}^{t}$ of $\widetilde{B}_{\xi}^{t}$ passing through $x_{0}$ is at Hausdorff distance $\lesssim t$ from $c$ in $\mathbb{R}^{2}$, there exists a flat strip $S_{t}$, parallel to $c$, of width $w_{t}$ with $w_{0}-w_{t} \lesssim t$, contained in $\widetilde{B}_{\xi}^{t}$, where $w_{0}$ is the width of $\widetilde{B}_{\xi}$. The straight lines foliating $S_{t}$ project to systolic loops of $\left(M, g_{t}\right)$. Therefore, the trajectories of $-\nabla_{g_{t}} \beta_{\xi}^{g_{t}}$ arising from the points of $S_{t}$ lie in these straight lines and so are parallel to $c$. As $c_{0}$ smoothly varies with $t$ (see [13] and the beginning of Section 12.1), we derive that

$$
\left\|d \beta_{\xi}^{g_{t}}-d \beta_{\xi}^{g_{0}}\right\|_{L^{\infty}\left(\tilde{\Delta} \cap S_{t}\right)} \lesssim t .
$$

On the other hand, the area of $\widetilde{\Delta} \backslash S_{t}$ is $\lesssim t$. Thus, from Proposition 12.2,

$$
\left\|d \beta_{\xi}^{g_{t}}-d \beta_{\xi}^{g_{0}}\right\|_{L^{2}\left(\tilde{\Delta} \backslash S_{t}\right)} \lesssim t \sqrt{t} .
$$

The desired estimate follows in this case too. Hence the result.

Remark 12.7 For any oriented simple closed geodesic $\gamma$ on $M_{0}$, we can define a oneparameter family of metrics $\left(g_{t}\right)$ by twisting the metric $g_{0}$ along $\gamma$. More precisely, cut $M_{0}$ open along $\gamma$ and glue the boundary components back together after making a twist of parameter $t$. The resulting metric on the surface, denoted by $g_{t}$, is piecewise flat with conical singularities of angles greater than $2 \pi$. (The conical singularities of $M_{0}$ may be split into two conical singularities in the process if $\gamma$ passes through some of them.) The proof of Proposition 12.4 can easily be modified to show that $\left(g_{t}\right)$ is a slow metric variation too.

Note that in the hyperbolic case, the metric variations obtained by twisting along simple closed geodesics induce a dense set of directions in the tangent space of the Teichmüller space at the initial metric. 


\section{References}

[1] J-C Álvarez Paiva, F Balacheff, Infinitesimal systolic rigidity of metrics all of whose geodesics are closed and of the same length arXiv:0912.3413

[2] F Balacheff, Sur la systole de la sphère au voisinage de la métrique standard, Geom. Dedicata 121 (2006) 61-71 MR2276235

[3] F Balacheff, A local optimal diastolic inequality on the two-sphere, J. Topol. Anal. 2 (2010) 109-121 MR2646992

[4] C Bavard, Inégalité isosystolique pour la bouteille de Klein, Math. Ann. 274 (1986) 439-441 MR842624

[5] C Bavard, Inégalités isosystoliques conformes pour la bouteille de Klein, Geom. Dedicata 27 (1988) 349-355 MR960206

[6] C Bavard, Inégalités isosystoliques conformes, Comment. Math. Helv. 67 (1992) 146-166 MR1144618

[7] G Besson, G Courtois, S Gallot, Entropies et rigidités des espaces localement symétriques de courbure strictement négative, Geom. Funct. Anal. 5 (1995) 731-799 MR1354289

[8] R L Bryant, On extremals with prescribed Lagrangian densities, from: "Manifolds and geometry (Pisa, 1993)", (P de Bartolomeis, F Tricerri, E Vesentini, editors), Sympos. Math. XXXVI, Cambridge Univ. Press (1996) 86-111 MR1410069

[9] D Burago, S Ivanov, On asymptotic volume of Finsler tori, minimal surfaces in normed spaces, and symplectic filling volume, Ann. of Math. (2) 156 (2002) 891-914 MR1954238

[10] D Burago, S Ivanov, Boundary rigidity and filling volume minimality of metrics close to a flat one, Ann. of Math. (2) 171 (2010) 1183-1211 MR2630062

[11] E Calabi, Extremal isosystolic metrics for compact surfaces, from: "Actes de la Table Ronde de Géométrie Différentielle (Luminy, 1992)”, (A L Besse, editor), Sémin. Congr. 1, Soc. Math. France (1996) 167-204 MR1427758

[12] C B Croke, M Katz, Universal volume bounds in Riemannian manifolds, from: "Surveys in differential geometry, Vol. VIII (Boston, MA, 2002)”, (S-T Yau, editor), Surv. Differ. Geom. VIII, Int. Press, Somerville, MA (2003) 109-137 MR2039987

[13] J Eells, L Lemaire, Deformations of metrics and associated harmonic maps, Proc. Indian Acad. Sci. Math. Sci. 90 (1981) 33-45 MR653945

[14] A El Soufi, S Ilias, Riemannian manifolds admitting isometric immersions by their first eigenfunctions, Pacific J. Math. 195 (2000) 91-99 MR1781616

[15] M Gromov, Filling Riemannian manifolds, J. Differential Geom. 18 (1983) 1-147 MR697984 
[16] M Gromov, Metric structures for Riemannian and non-Riemannian spaces, Progress in Math. 152, Birkhäuser, Boston (1999) MR1699320 Based on the 1981 French original [Textes Math. 1 MR0682063], With appendices by M Katz, P Pansu and S Semmes, Translated from the French by S M Bates

[17] C Horowitz, K Usadi Katz, M G Katz, Loewner's torus inequality with isosystolic defect, J. Geom. Anal. 19 (2009) 796-808 MR2538936

[18] S V Ivanov, On two-dimensional minimal fillings, Algebra i Analiz 13 (2001) 26-38 MR1819361 In Russian

[19] M G Katz, Systolic geometry and topology, Math. Surveys and Monogr. 137, Amer. Math. Soc. (2007) MR2292367 With an appendix by J P Solomon

[20] M G Katz, S Sabourau, Entropy of systolically extremal surfaces and asymptotic bounds, Ergodic Theory Dynam. Systems 25 (2005) 1209-1220 MR2158402

[21] M G Katz, S Sabourau, Hyperelliptic surfaces are Loewner, Proc. Amer. Math. Soc. 134 (2006) 1189-1195 MR2196056

[22] M G Katz, S Sabourau, An optimal systolic inequality for CAT(0) metrics in genus two, Pacific J. Math. 227 (2006) 95-107 MR2247874

[23] N Nadirashvili, Berger's isoperimetric problem and minimal immersions of surfaces, Geom. Funct. Anal. 6 (1996) 877-897 MR1415764

[24] P M Pu, Some inequalities in certain nonorientable Riemannian manifolds, Pacific J. Math. 2 (1952) 55-71 MR0048886

[25] S Sabourau, Systoles des surfaces plates singulières de genre deux, Math. Z. 247 (2004) 693-709 MR2077416

[26] S Sabourau, Local extremality of the Calabi-Croke sphere for the length of the shortest closed geodesic, J. Lond. Math. Soc. (2) 82 (2010) 549-562 MR2739055

[27] M Troyanov, Prescribing curvature on compact surfaces with conical singularities, Trans. Amer. Math. Soc. 324 (1991) 793-821 MR1005085

Laboratoire de Mathématiques et Physique Théorique, Université François-Rabelais Tours Parc de Grandmont, 37200 Tours, France

sabourau@lmpt .univ-tours.fr

http://www. Impt.univ-tours.fr/ sabourau

Proposed: Dmitri Burago

Seconded: Tobias H. Colding, Leonid Polterovich
Received: 4 March 2010

Revised: 26 June 2011 